\title{
Electrocatalytic oxidative cleavage of electronegatively substituted stilbenes in acetonitrile-water employing a new high-oxidation-potential electrocatalyst. An electrochemical equivalent of ozonolysis.
}

\author{
Xin Wu, Anthony P. Davis, and Albert J. Fry* \\ Chemistry Department, Wesleyan University, Middletown, CT 06459
}

\section{SUPPORTING INFORMATION}

\section{Table of Contents}

General Information

Electrolysis Setup

Representative Electrolysis

Preparation of Non-commercial Starting Materials

Synthesis of 4-Formyl-3',5'-bis(trifluoromethyl)stilbenes (E/Z) (2c)

Synthesis of 4-Cyano-3',5'-bis(trifluoromethyl)stilbenes (E/Z) (2d)

Synthesis of (E)-4-Formyl-4'-trifluoromethylstilbene (2e)

${ }^{1} \mathrm{H}$ NMR spectra

${ }^{13} \mathrm{C}$ NMR spectra 
General Information: NMR was obtained using a Varian Mercury Vx $300 \mathrm{MHz}$ NMR spectrometer using VNMR v. 5.3 software. Chemical shifts are reported in parts per million (ppm) with the solvent used as the internal standard; $\mathrm{CD}_{3} \mathrm{CN}$ : $\delta 1.94 \mathrm{ppm}(5)\left({ }^{1} \mathrm{H}\right.$ NMR); $\delta 1.39$ ppm (7) ( ${ }^{13}$ C NMR). An HP 5890 Series II GC with an HP-5MS column coupled to an HP 5972 Series MS Detector was used to monitor electrolyses. An Agilent Technologies $6890 \mathrm{~N}$ Gas Chromatograph with an HP-5 column coupled to a 5973N MS Detector was used for compound characterization. All HRMS were conducted by the Mass Spectroscopy Lab at the University of Illinois, Urbana-Champagne. Microanalyses were conducted by Chemisar Laboratories, Inc., Ontario, Canada.

Electrolysis Setup: Controlled-potential electrolyses were carried out at room temperature in a $20-\mathrm{mL}$ test tube equipped with magnetic stir bar (Figure S1), coulometer, and circuitry arranged such that electrolysis was allowed to proceed for alternating two-minute "on" and "off" periods. The electrolyte solution consisted of 5-10 $\mathrm{mL}$ of a $0.1 \mathrm{M} \mathrm{LiClO}_{4}$ solution in acetonitrile. The working electrode consisted of a $1 \mathrm{~cm}$ $\times 3 \mathrm{~cm}$ piece of woven graphite fiber carbon cloth (Union Carbide WCA graphite cloth). The counter electrode was a $2.5 \mathrm{~cm} \times 2.5 \mathrm{~cm}$ platinum gauze electrode folded to save space. Electrical contact to each electrode was made by a $15-\mathrm{cm}$ platinum wire. The reference electrode consisted of a silver wire fitted into a piece of heavy-wall Teflon tubing sealed at the bottom with a porous Vycor plug and containing a $0.1 \mathrm{M} \mathrm{AgNO}_{3}$ solution in acetonitrile.

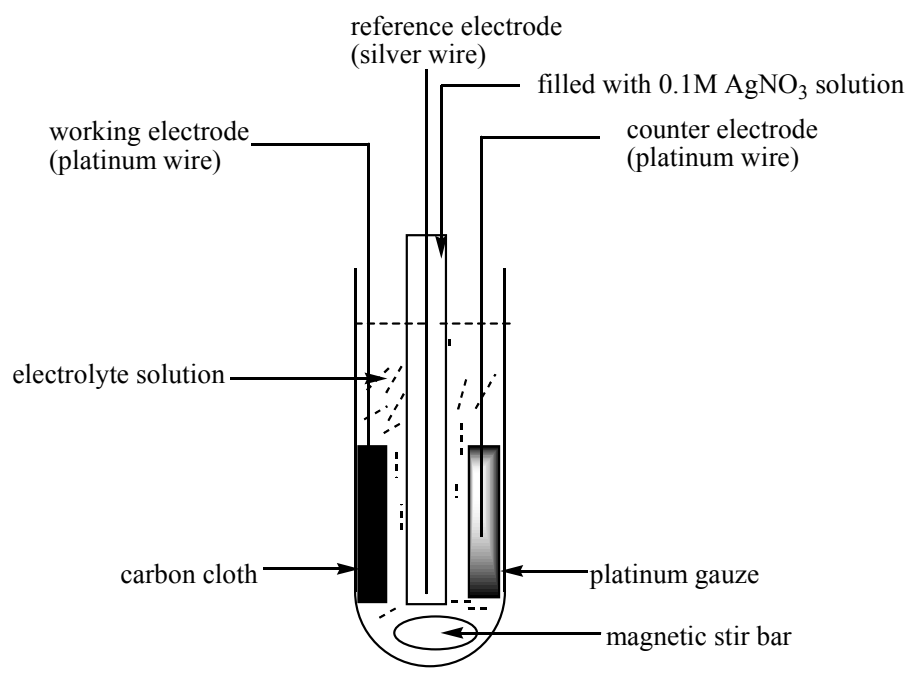

Figure S1. Diagram of experimental electrolysis apparatus. 
Representative Electrolysis - Electrocatalytic oxidation of 4,4'-bis(trifluoromethyl)stilbene (2a): 4,4'-bis(trifluoromethyl)stilbene, 2a, (70 mg, $0.22 \mathrm{mmol}$ ) and 4,4',4'trimethyl-2,2',2"-trinitrotriphenylamine, $1 \mathbf{c},(12 \mathrm{mg}, 0.028 \mathrm{mmol})$ were added to $10 \mathrm{~mL}$

of electrolyte solution in the electrolysis cell. A drop of water was added to the solution, and the electrolysis was conducted at $+1.30 \mathrm{~V} v$ s. the $\mathrm{Ag} / \mathrm{AgNO}_{3}$ reference. Sampling and GC-MS analysis at regular intervals showed the gradual conversion of 2a into 4trifluoromethylbenzaldehyde. After 17 hours, the electrolysis current had decayed from $11 \mathrm{~mA}$ to a constant $3 \mathrm{~mA}$, and GC-MS showed $100 \%$ conversion of $2 \mathbf{a}$ to aldehyde. A total of 128 coulombs ( 0.00133 faradays) had been consumed, corresponding to an uptake of 6.03 faradays per mole of 2a. The solution was washed with $20 \mathrm{~mL}$ of water and then extracted with dichloromethane $(2 \times 20 \mathrm{~mL})$. The combined organic extracts were dried over $\mathrm{Na}_{2} \mathrm{SO}_{4}$, evaporated, and chromatographed over silica gel to afford pure 4-trifluoromethylbenzaldehyde ( $73 \mathrm{mg}, 96 \%$ yield).

\section{Preparation of Non-commercial Starting Materials:}

\section{a) Synthesis of (4-Trifluoromethylbenzyl)triphenylphosphonium bromide: 4-Tri-} fluoromethylbenzyl bromide (1.20 g, $5 \mathrm{mmol})$ and triphenylphosphine $(1.84 \mathrm{~g}, 7 \mathrm{mmol})$ were mixed in $80 \mathrm{~mL}$ of benzene and refluxed for 16 hours, during which time a white precipitate formed. The suspension was cooled to room temperature and vacuumfiltered, and then the white solid product was washed with cold petroleum ether $(3 \times 15$ $\mathrm{mL}$ ). The desired product was collected in $75 \%$ yield and used directly for the synthesis of 4-formyl-4'-trifluoromethylstilbene.

\section{b) Synthesis of (3,5-Bis(trifluoromethyl)benzyl)triphenylphosphonium bromide:} 3,5-Bis(trifluoromethyl)benzyl bromide $(1.0 \mathrm{~g}, 3.3 \mathrm{mmol})$ and triphenylphosphine $(1.20$ $\mathrm{g}, 1.4 \mathrm{eq}$ ) were mixed in $20 \mathrm{~mL} \mathrm{CH}_{2} \mathrm{Cl}_{2}$ and refluxed overnight, during which time a white precipitate formed. Removed solvent by rotary evaporation, and washed solid products with diethyl ether. The desired product was collected in $97 \%$ yield and used directly for the syntheses of the 4-cyano- and the 4-formyl-3',5'-bis(trifluoromethyl)stilbenes. 
Synthesis of 4-cyano-3',5'-bis(trifluoromethyl)stilbenes (E/Z) (2c): 3,5-Bis(trifluoromethyl)benzyltriphenylphosphonium bromide (569 mg, $1.0 \mathrm{mmol})$, 4-cyanobenzaldehyde (131 mg, $1.0 \mathrm{mmol}$ ), tetrabutylammonium iodide (45 mg, $0.12 \mathrm{eq})$, and $\mathrm{Na}_{2} \mathrm{CO}_{3}(5.00 \mathrm{~g})$ were mixed dry in a round-bottom flask and then stirred upon addition of $10 \mathrm{~mL} \mathrm{CH} \mathrm{Cl}_{2}$ and $10 \mathrm{~mL}$ dist. $\mathrm{H}_{2} \mathrm{O}$. After stirring overnight, extracted with $\mathrm{CH}_{2} \mathrm{Cl}_{2}(3 \times 10 \mathrm{~mL})$, washed combined organic layers with brine, dried over $\mathrm{Na}_{2} \mathrm{SO}_{4}$, and removed solvent by rotary evaporation. Purified over silica gel by repeated flash chromatography $(10 \%$ EtOAc in hexanes); recovered a yellowish liquid that slowly crystallized (160 mg, (Z)-4cyano-3',5'-bis(trifluoromethyl)stilbene and a white solid (70 mg, (E)-4-cyano-3',5'-bis(trifluoromethyl)stilbene). Combined yield was $67 \%$. In the ${ }^{13} \mathrm{C}$ NMR spectra, only ten of twelve peaks appeared in 4000 scans; 12,000 scans and addition of $\mathrm{Cr}(\mathrm{acac})_{3}$ resulted in fifteen peaks. However, two of the fifteen peaks ( $\sim 123$ and $\sim 126 \mathrm{ppm})$ were common in all ${ }^{13} \mathrm{C}$ spectra, suggesting a trace impurity in the solvent or the $\mathrm{Cr}(\mathrm{acac})_{3}$, and one other peak ( 116 ppm) was common only in $E$ - and Z-4-cyano-3',5'-bis(trifluoromethyl)stilbene, suggesting possibly another trace impurity in this reaction.

- (E)-4-cyano-3',5'-bis(trifluoromethyl)stilbene: Mp 194-197 C. ${ }^{1} \mathrm{H}$ NMR $\left(\mathrm{CD}_{3} \mathrm{CN}, 300 \mathrm{MHz}\right): \delta(\mathrm{ppm})=8.16(\mathrm{~s}, 2 \mathrm{H}), 7.92(\mathrm{~s}, 1 \mathrm{H}), 7.75(\mathrm{~d}, J=1.5 \mathrm{~Hz}, 4 \mathrm{H})$, 7.49/7.45 (q $\left.\mathrm{q}_{\mathrm{AB}}, J=16.4 \mathrm{~Hz}, 2 \mathrm{H}\right) .{ }^{13} \mathrm{C} \mathrm{NMR}\left(300 \mathrm{MHz}, \mathrm{CD}_{3} \mathrm{CN}, \mathrm{Cr}(\mathrm{acac})_{3}\right)$ : $\delta(\mathrm{ppm})=142.0,140.3,133.8,132.8,132.3,131.9,130.0,128.5,128.1(\mathrm{q}), 126.4$, 122.8, 122.5, 119.7, 115.9, 112.3. MS: $m / z$ (\% of base peak): 341(100, $\left.\mathrm{M}^{+}\right)$, 322(15), 301(7), 272(15), 271(15), 252(26), 203(23). Anal. Calcd for $\mathrm{C}_{17} \mathrm{H}_{9} \mathrm{~F}_{6} \mathrm{~N}$ : C, 59.83; H, 2.66. Found: C, 60.11; H, 3.06. HRMS (CI 70 eV): Calcd, 341.0635; Found, 341.0639.

- (Z)-4-cyano-3',5'-bis(trifluoromethyl)stilbene: Mp $62^{\circ}-63^{\circ} \mathrm{C}$. ${ }^{1} \mathrm{H} \mathrm{NMR}$ $\left(\mathrm{CD}_{3} \mathrm{CN}, 300 \mathrm{MHz}\right): \delta(\mathrm{ppm})=7.86(\mathrm{~s}, 1 \mathrm{H}), 7.72(\mathrm{~s}, 2 \mathrm{H}), 7.61(\mathrm{~d}, J=8.4 \mathrm{~Hz}, 2 \mathrm{H})$, $7.34(\mathrm{~d}, J=8.4 \mathrm{~Hz}, 2 \mathrm{H}), 6.91 / 6.87(\mathrm{q} \mathrm{AB}, J=12.3 \mathrm{~Hz}, 2 \mathrm{H}) .{ }^{13} \mathrm{C} \mathrm{NMR}(300 \mathrm{MHz}$, $\left.\mathrm{CD}_{3} \mathrm{CN}, \mathrm{Cr}(\mathrm{acac})_{3}\right): \delta(\mathrm{ppm})=141.9,139.8,133.4,133.0,132.3,131.8,130.7$, 130.6, 130.4(q), 126.2, 122.6, 122.0, 116.1, 119.5, 111.9 MS: $m / z$ (\% of base peak): 341(100, $\left.\mathrm{M}^{+}\right)$, 322(15), 301(7), 272(15), 271(15), 252(26), 203(23). Anal. Calcd for $\mathrm{C}_{17} \mathrm{H}_{9} \mathrm{~F}_{6} \mathrm{~N}$ : C, 59.83; H, 2.66. Found: C, 59.68; H, 2.62. HRMS (CI 70 eV): Calcd, 341.0635; Found, 341.0639. 
Synthesis of 4-formyl-3',5'-bis(trifluoromethyl)stilbenes (E/Z) (2d): 3,5-Bis(trifluoromethyl)benzyltriphenylphosphonium bromide (569 mg, $1.0 \mathrm{mmol})$, terephthalaldehyde (134 mg, $1.0 \mathrm{mmol}$ ), tetrabutylammonium iodide (45 mg, $0.12 \mathrm{eq}$ ), and $\mathrm{Na}_{2} \mathrm{CO}_{3}(5.00 \mathrm{~g}$ ) were mixed dry in a round-bottom flask and then stirred upon addition of $10 \mathrm{~mL} \mathrm{CH}_{2} \mathrm{Cl}_{2}$ and $10 \mathrm{~mL}$ dist. $\mathrm{H}_{2} \mathrm{O}$. After stirring overnight, extracted with $\mathrm{CH}_{2} \mathrm{Cl}_{2}(3 \times 10 \mathrm{~mL})$, washed combined organic layers with brine, dried over $\mathrm{Na}_{2} \mathrm{SO}_{4}$, and removed solvent by rotary evaporation. Purified over silica gel by flash chromatography (10\% EtOAc / hexanes); recovered ca. $220 \mathrm{mg}$ of product (64\% yield). GC-MS analysis indicated a 60/40 mixture of (E/Z) diastereomers. Partially separated diastereomers over silica gel by flash chromatography (3:2 $\mathrm{CH}_{2} \mathrm{Cl}_{2} /$ hexanes); recovered a yellowish liquid (49 $\mathrm{mg},(Z)$ 4-formyl-3',5'-bis(trifluoromethyl)stilbene); a white solid (21 mg, (E)-4-formyl-3',5'bis(trifluoromethyl)stilbene; and the remaining (E/Z) mixture. In the ${ }^{13} \mathrm{C}$ NMR spectra, 4000 scans and addition of $\mathrm{Cr}(\mathrm{acac})_{3}$ resulted in fourteen peaks. However, two of the fourteen peaks $(\sim 123$ and $\sim 126 \mathrm{ppm})$ were common in all ${ }^{13} \mathrm{C}$ spectra, suggesting a trace impurity in the solvent or the $\mathrm{Cr}(\mathrm{acac})_{3}$.

- (E)-4-formyl-3',5'-bis(trifluoromethyl)stilbene: Mp $140^{\circ}-142^{\circ} \mathrm{C} .{ }^{1} \mathrm{H} \mathrm{NMR}$ $\left(\mathrm{CD}_{3} \mathrm{CN}, 300 \mathrm{MHz}\right): \delta(\mathrm{ppm})=10.0(\mathrm{~s}, \mathrm{CHO}), 8.18(\mathrm{~s}, 2 \mathrm{H}), 7.93(\mathrm{~d}, J=8.7 \mathrm{~Hz}$, 2H), $7.92(\mathrm{~s}, 1 \mathrm{H}), 7.79(\mathrm{~d}, J=8.1 \mathrm{~Hz}, 2 \mathrm{H}), 7.53 / 7.47\left(\mathrm{q}_{\mathrm{AB}}, J=16.2 \mathrm{~Hz}, 2 \mathrm{H}\right) .{ }^{13} \mathrm{C}$ NMR (300 MHz, $\left.\mathrm{CD}_{3} \mathrm{CN}, \mathrm{Cr}(\mathrm{acac})_{3}\right): \delta(\mathrm{ppm})=192.4(\mathrm{CHO}), 142.7,139.9,136.7$, $132.1,131.8,131.7,130.5,129.1,127.9,127.5,125.8,122.2,121.7$. MS: $m / z(\%$ of base peak): 344(100, $\left.\mathrm{M}^{+}\right), 343(100), 325(15), 315(14), 295(29), 275(29)$, 246(85), 225(23). Anal. Calcd for $\mathrm{C}_{17} \mathrm{H}_{10} \mathrm{~F}_{6} \mathrm{O}: \mathrm{C}, 59.31$; H, 2.93. Found: C, 59.35; H, 3.00. HRMS (CI 70 eV): Calcd, 344.0635; Found, 344.0636.

- (Z)-4-formyl-3',5'-bis(trifluoromethyl)stilbene: Yellow liquid. ${ }^{1} \mathrm{H}$ NMR $\left(\mathrm{CD}_{3} \mathrm{CN}, 300 \mathrm{MHz}\right): \delta(\mathrm{ppm})=9.95(\mathrm{~s}, \mathrm{CHO}), 7.85(\mathrm{~s}, 1 \mathrm{H}), 7.78(\mathrm{~d}, J=8.1 \mathrm{~Hz}$, $2 \mathrm{H}), 7.74(\mathrm{~s}, 2 \mathrm{H}), 7.39(\mathrm{~d}, J=8.1 \mathrm{~Hz}, 2 \mathrm{H}), 6.95 / 6.87\left(\mathrm{q}_{\mathrm{AB}}, J=12.4 \mathrm{~Hz}, 2 \mathrm{H}\right) .{ }^{13} \mathrm{C}$ NMR (300 MHz, $\left.\mathrm{CD}_{3} \mathrm{CN}, \mathrm{Cr}(\mathrm{acac})_{3}\right): \delta(\mathrm{ppm})=193.0(\mathrm{CHO}), 143.2,140.1,136.8$, 133.6, 132.3, 131.8, 130.7, 130.5, 130.4, 130.3, 126.2, 122.6, 122.0. MS: $m / z(\%$ of base peak): 344(100, $\left.\mathrm{M}^{+}\right), 343(100), 325(15), 315(14), 295(29), 275(29)$, 246(85), 225(23). Anal. Calcd for $\mathrm{C}_{17} \mathrm{H}_{10} \mathrm{~F}_{6} \mathrm{O}: \mathrm{C}, 59.31$; H, 2.93. Found: C, 59.04; H, 2.76. HRMS (CI 70 eV): Calcd, 344.0635; Found, 344.0636. 
Synthesis of (E)-4-formyl-4'-trifluoromethylstilbene (2e): 4-(Trifluoromethylbenzyl)triphenylphosphonium bromide (1.525 g, $3.2 \mathrm{mmol})$, terephthalaldehyde (429 mg, 3.2 mmol), tetrabutylammonium iodide (45 mg, $0.12 \mathrm{eq}$ ), and $\mathrm{Na}_{2} \mathrm{CO}_{3}(5.00 \mathrm{~g}$ ) were mixed dry in a round-bottom flask and then stirred upon addition of $10 \mathrm{~mL} \mathrm{CH}_{2} \mathrm{Cl}_{2}$ and $10 \mathrm{~mL}$ dist. $\mathrm{H}_{2} \mathrm{O}$. After stirring overnight, extracted with $\mathrm{CH}_{2} \mathrm{Cl}_{2}(3 \times 10 \mathrm{~mL})$, washed combined organic layers with brine, dried over $\mathrm{Na}_{2} \mathrm{SO}_{4}$, and removed solvent by rotary evaporation. Purified over silica gel by flash chromatography (10\% EtOAc in hexanes); crystallized in EtOH $(95 \%)$ to yield thin, white crystals. The $(Z)$ - diastereomer was not isolated. In the ${ }^{13} \mathrm{C}$ NMR spectra, 2000 scans and addition of $\mathrm{Cr}(\mathrm{acac})_{3}$ resulted in fourteen peaks. However, two of the fourteen peaks $(\sim 123$ and $\sim 126 \mathrm{ppm})$ were common in all ${ }^{13} \mathrm{C}$ spectra, suggesting a trace impurity in the solvent or the $\mathrm{Cr}(\mathrm{acac})_{3} . \mathrm{Mp}: 128^{\circ}-130^{\circ} \mathrm{C} .{ }^{1} \mathrm{H}$ $\operatorname{NMR}\left(\mathrm{CD}_{3} \mathrm{CN}, 300 \mathrm{MHz}\right): \delta(\mathrm{ppm})=9.99(\mathrm{~s}, \mathrm{CHO}), 7.91(\mathrm{~d}, J=6.3 \mathrm{~Hz}, 2 \mathrm{H}), 7.78(\mathrm{~d}, J=$ $8.1 \mathrm{~Hz}, 4 \mathrm{H}), 7.71(\mathrm{~d}, J=8.7 \mathrm{~Hz}, 2 \mathrm{H}), 7.43(\mathrm{~s}, 2 \mathrm{H}) .{ }^{13} \mathrm{C} \mathrm{NMR}\left(\mathrm{CD}_{3} \mathrm{CN}, 300 \mathrm{MHz}\right)$ : $\delta(\mathrm{ppm})=193.0(\mathrm{CHO}), 143.7,141.7,137.0,131.2,131.1,131.0,130.2,129.8,128.33$, 128.30, 127.2, 126.7(q), 123.6. MS: $m / z$ (\% of base peak): 276(100, $\left.\mathrm{M}^{+}\right), 275(36)$, 257(8), 247(19), 227(23), 207(10), 179(31), 178(65). Anal. Calcd for $\mathrm{C}_{16} \mathrm{H}_{11} \mathrm{~F}_{3} \mathrm{O}: \mathrm{C}$, 69.56; H, 4.01. Found: C, 69.29; H, 4.29. HRMS (CI 70 eV): Calcd, 276.0760; Found, 276.0762 . 
E-4-CN-3, $5^{*} m \mathrm{biscF} 3 \mathrm{st}$ i l bene

Archlve directory: /export/hoine/vnmr2/vnmesys/data

Pulse Sequence: s2pul

Solvent: $C 03 \mathrm{CN}$

Fife: APD 4 CN3 $298.1 \mathrm{~K}$.

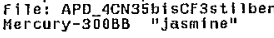

Re lax. delay $1.000 \mathrm{sec}$

Acq. time $1.997 \mathrm{sec}$

Width $4803,1 \mathrm{~Hz}$

OBSERVE H1, 300.0546289 $\mathrm{MHZ}$

DATA PROCESSIN

Total time o min, $25 \mathrm{sec}$

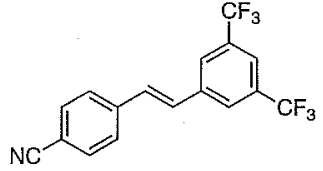

2C (E)

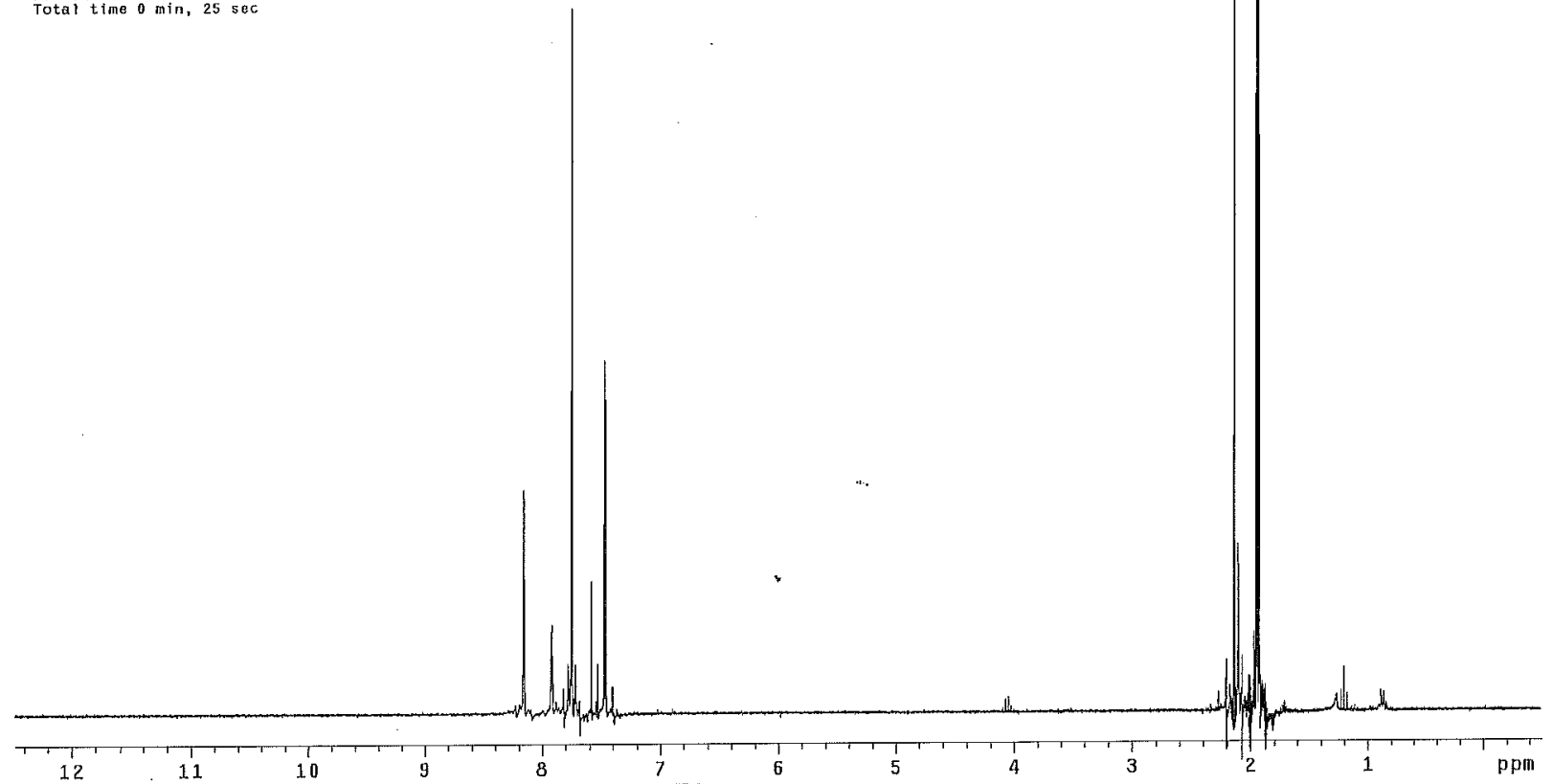

Figure S2. Full ${ }^{1} \mathrm{H}$ NMR spectrum of $2 \mathrm{c}$ (E) $\left(300 \mathrm{MHz}, \mathrm{CD}_{3} \mathrm{CN}\right)$.

E $\rightarrow 4-\mathrm{CN}-3^{\prime}, 5^{2}-b 15 \mathrm{CF} 3 \mathrm{~s}$ t1 libene

Fllename:_AuH

Archive diroctory: /export/home/ynmrz/unmrsys/data

Pulse Sequence: 52pur

SoTvent: $\mathrm{CD} 3 \mathrm{CN}$

Temp: $25.0 \mathrm{C}, 298.1 \times$

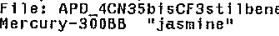

Relax. de lay 1.000 sec

Acq. $t$ 19le 1.997 sec

widin $4803.1 \mathrm{~Hz}$

8 repet It HONS
OBSERE H1, $300.0546289 \mathrm{MHZ}$
DATA PROCESSING

FT 5 tze 32768
Total tima 0 min, $25 \mathrm{sec}$

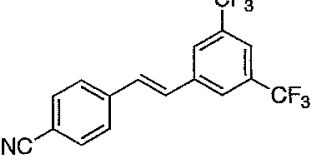

2c (E)

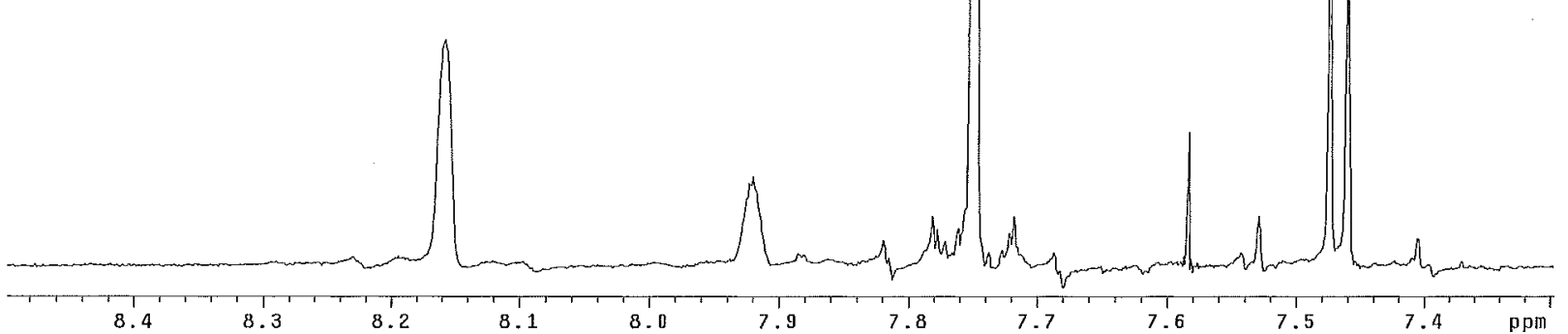

Figure S3. Partial ${ }^{\mathbf{H}} \mathrm{H}$ NMR spectrum of $2 \mathrm{c}$ (E) (300 $\left.\mathrm{MHz}, \mathrm{CD}_{3} \mathrm{CN}\right)$ 
z-4-CN-3, , 5,-bisch3stijlbene

Archive directory: /export/home/Ynar2/vnmrsys/data
Sample directory:

Pulse Sequence: s2pul

Solvent: CD3CN

Fieng. $25.0 \mathrm{C}$ C $298.1 \mathrm{~K}$

Relax-delay $1.000 \mathrm{sec}$

Acq time 1 im 997 soc

8 repetitions

DATA PROCESSING

FT size 32768
Total time 0 min, 25 sec
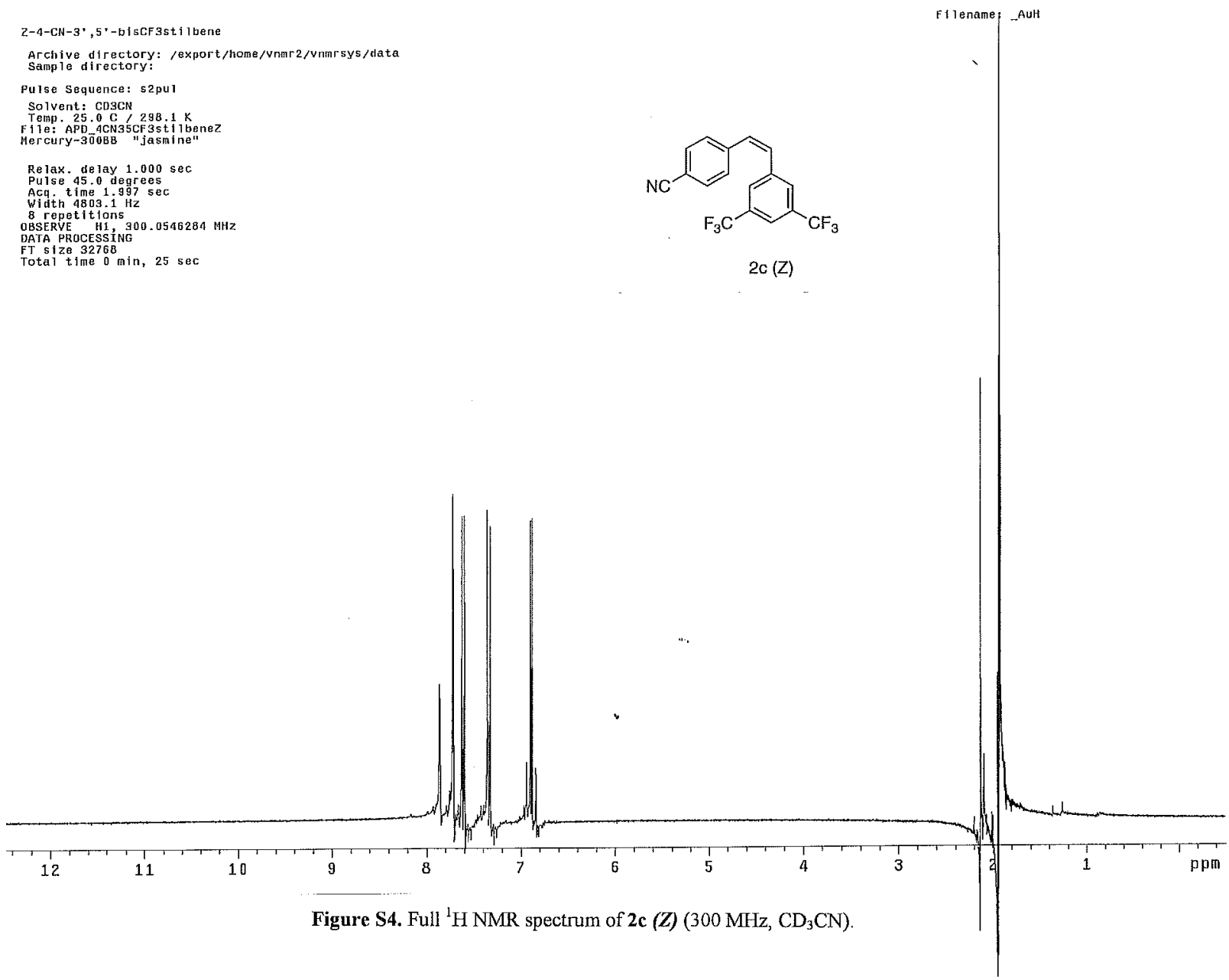

2-4-CN-3, , 5'-biscrasti i ibene

Archive directory: /export/home/vrar 2/vmirsys/data

Pulse Sequence: s2pul

Solvent: CDSCN

Fi ear : $25.00 \mathrm{C}$ ' $298.1 \mathrm{X}$

Relax.

ACq. $t$ 1me 1 . 997 sec

s ropotition

OBSERVE H1, 300.0546284 MHZ

Totatize 32768 ine $25 \mathrm{se}$
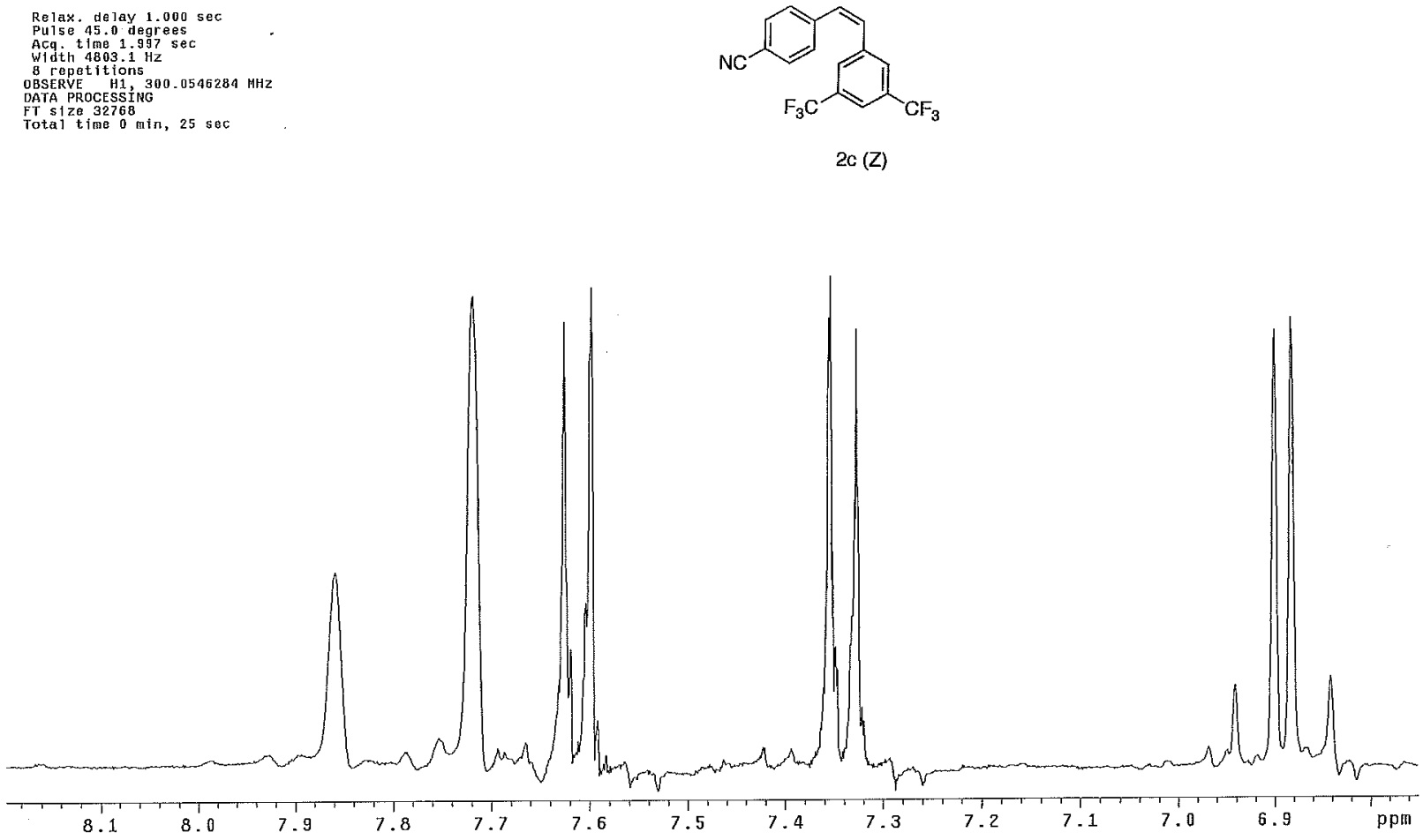

Figure S5. Partial ${ }^{1} \mathrm{H}$ NMR spectrum of $2 \mathrm{c}(Z)\left(300 \mathrm{MHz}, \mathrm{CD}_{3} \mathrm{CN}\right)$

$$
\text { S-8 }
$$




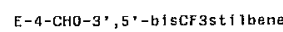

Archlve directory: /export/hoae/vnisr2/vnnirsys/data
Sample directory:

Pulse Sequence: s2pu

Solvent: $\mathrm{CD} 3 \mathrm{CN}$

Temp- $25.0 \mathrm{C}$ C $298.1 \mathrm{~K}$

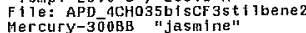

Relax. delay $1.000 \mathrm{sec}$

ACq $t$ tho

Acq
Widthe 4803.1997
Gith

8 repet it 1ons
OBSERVE H1,300.0546245 $\mathrm{MHz}$

DATA PROCESSÍNG

FT size 32758 in $25 \mathrm{sec}$

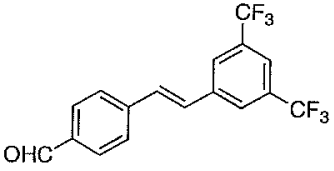

$2 d(E)$

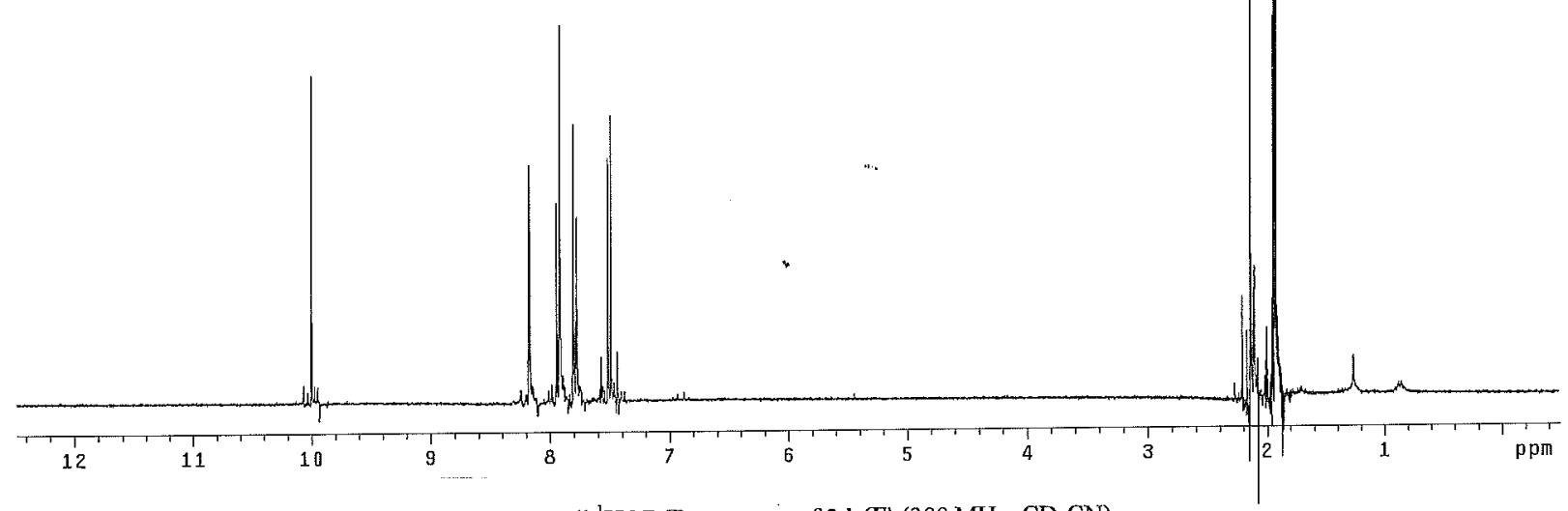

Figure S6. Full ${ }^{1} \mathrm{H}$ NMR spectrum of $2 \mathbf{d}(E)\left(300 \mathrm{MHz}, \mathrm{CD}_{3} \mathrm{CN}\right)$.

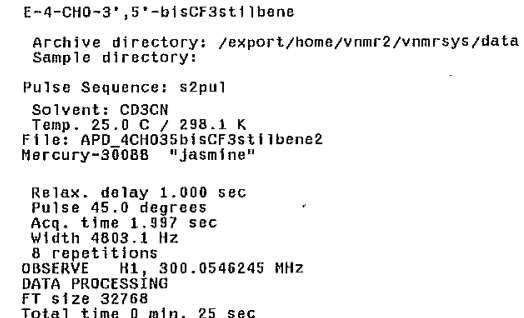

Total time $0 \mathrm{~min}, 25 \mathrm{sec}$
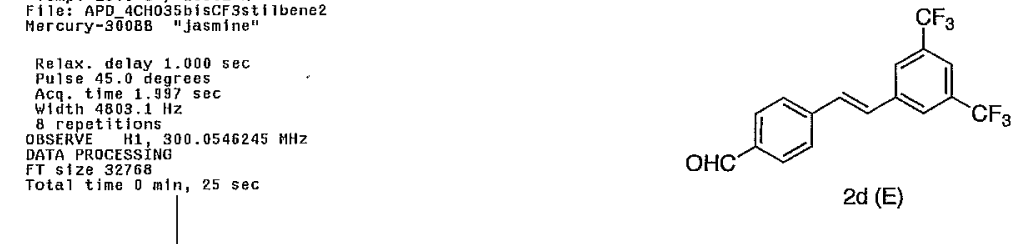

F1lename: _AuH

(u) $u$

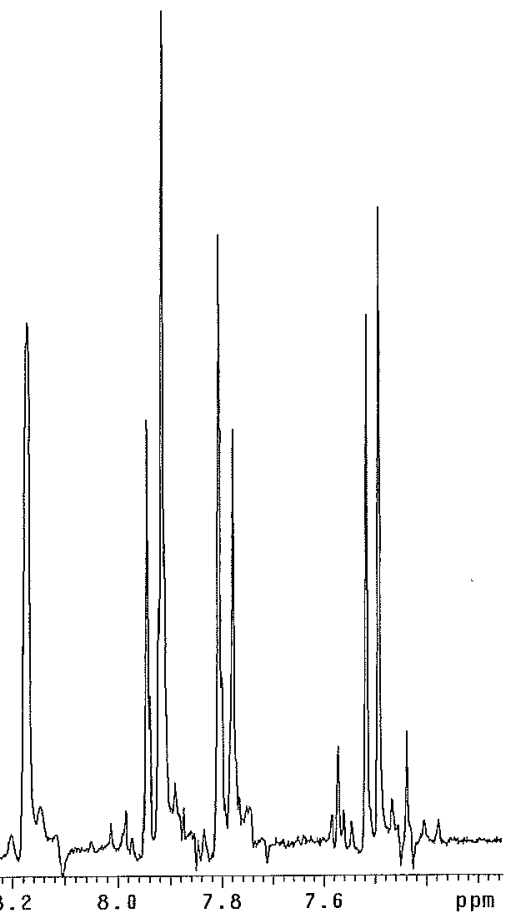

Figure S7. Partial ${ }^{\mathrm{l}} \mathrm{H} \mathrm{NMR}$ spectrum of $2 \mathbf{d}(E)\left(300 \mathrm{MHz}, \mathrm{CD}_{3} \mathrm{CN}\right)$. 
Archive directory: lexport/home/vnmr2/vnarsys/data
Sample directory:

Palse Sequence: s2pu

Tenp. $25.0 \mathrm{C} / 298.1 \mathrm{~K}$

ercury-30̃ozB "jasmine"

Relax. delay 1.000 se

Acq time $1.997 \mathrm{sec}$

Width 4 ing

OBSERVE H1 300.0546269 MHZ

Tot size 32768
Total time 0 inin, 25 sec

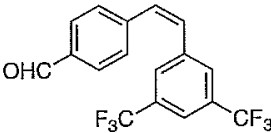

$2 d(Z)$

Figure S8. Full ${ }^{1} \mathrm{H}$ NMR spectrum of $2 \mathrm{~d}(Z)\left(300 \mathrm{MHz}, \mathrm{CD}_{3} \mathrm{CN}\right)$.

Z-4-CHO-3,, $5^{2}-6$ iscF3stimene

Fliename: _AuH

Archlve directory: /export/hoile/vnar2/vnmrsys/tata

Pulse Sequence: 82 pul

Solvent: CDSCN

File: APD 4CHO35biscF3st $\nmid$ ben

Ferax

Relax. delay 1.000

ACq. time 1,9975

8 repetitions
OBSERVE H1 $300.0546269 \mathrm{MHZ}$

FT size 32768
Total time 0 min, $25 \mathrm{sec}$
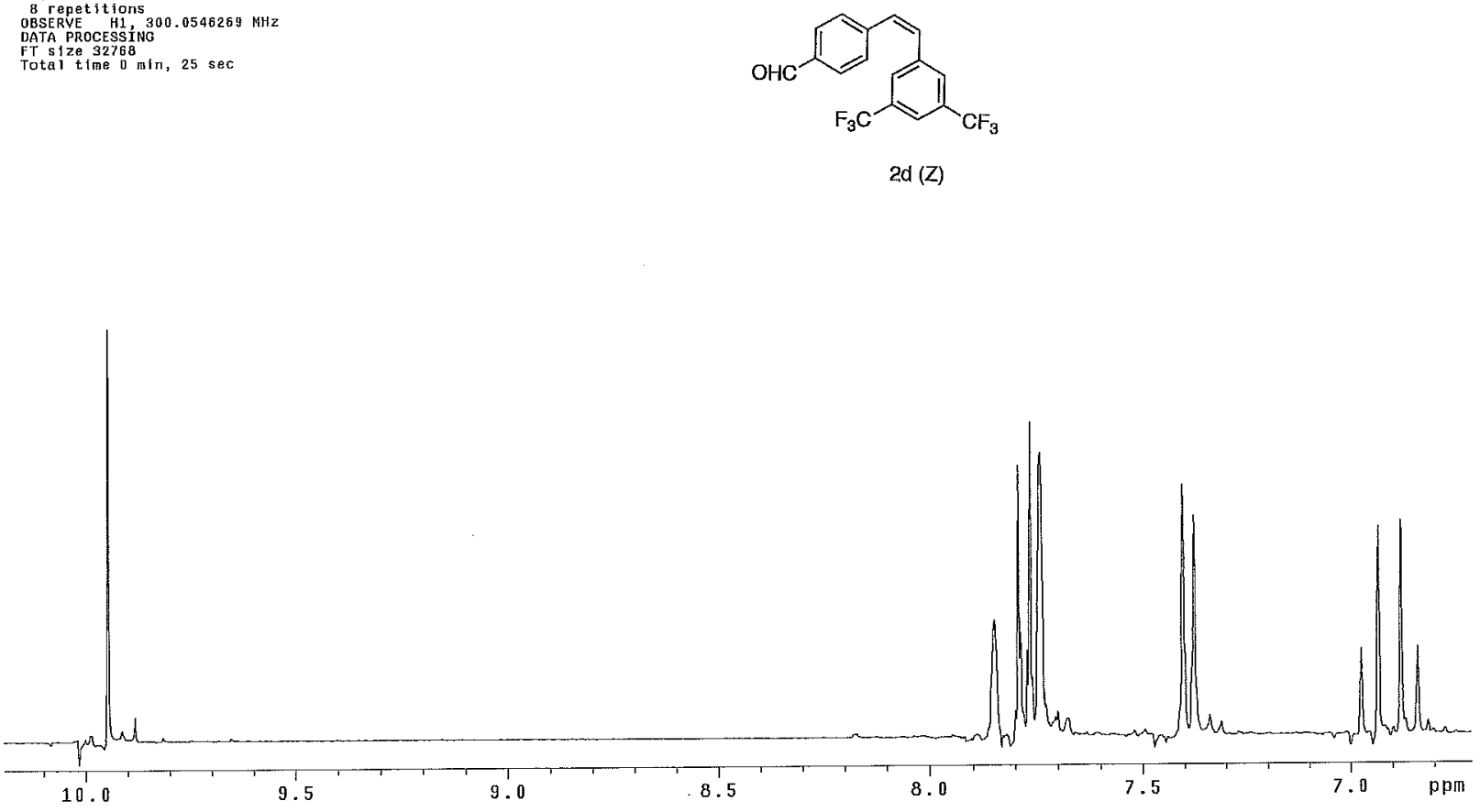

Figure S9. Partial ${ }^{1} \mathrm{H}$ NMR spectrum of $2 \mathrm{~d}(Z)\left(300 \mathrm{MHz}, \mathrm{CD}_{3} \mathrm{CN}\right)$

$$
\text { S-10 }
$$


4-Cho-4.-CF3stilbene

Archlve directory:
Sample directory:

Pulse Sequence: s2pul

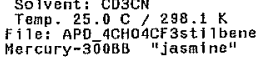

Relax. delay $1.000 \mathrm{sec}$

Acq $t$. degrees

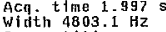

$B$ repetitions
OBSERVE H1, $300.0546243 \mathrm{MHZ}$

DATA PROCESSING

Total time 0 min, $25 \mathrm{sec}$

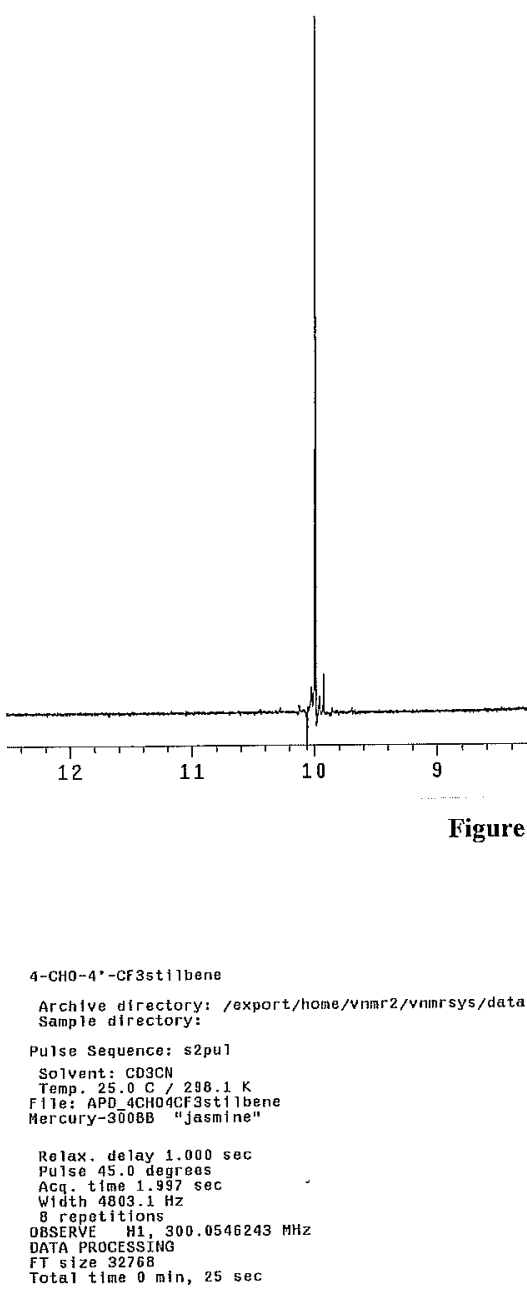

Figure S10. Full ${ }^{1} \mathrm{H}$ NMR spectrum of $2 \mathrm{e}\left(300 \mathrm{MHz}, \mathrm{CD}_{3} \mathrm{CN}\right)$

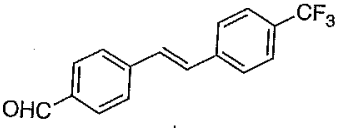

$2 e$
$2 e$

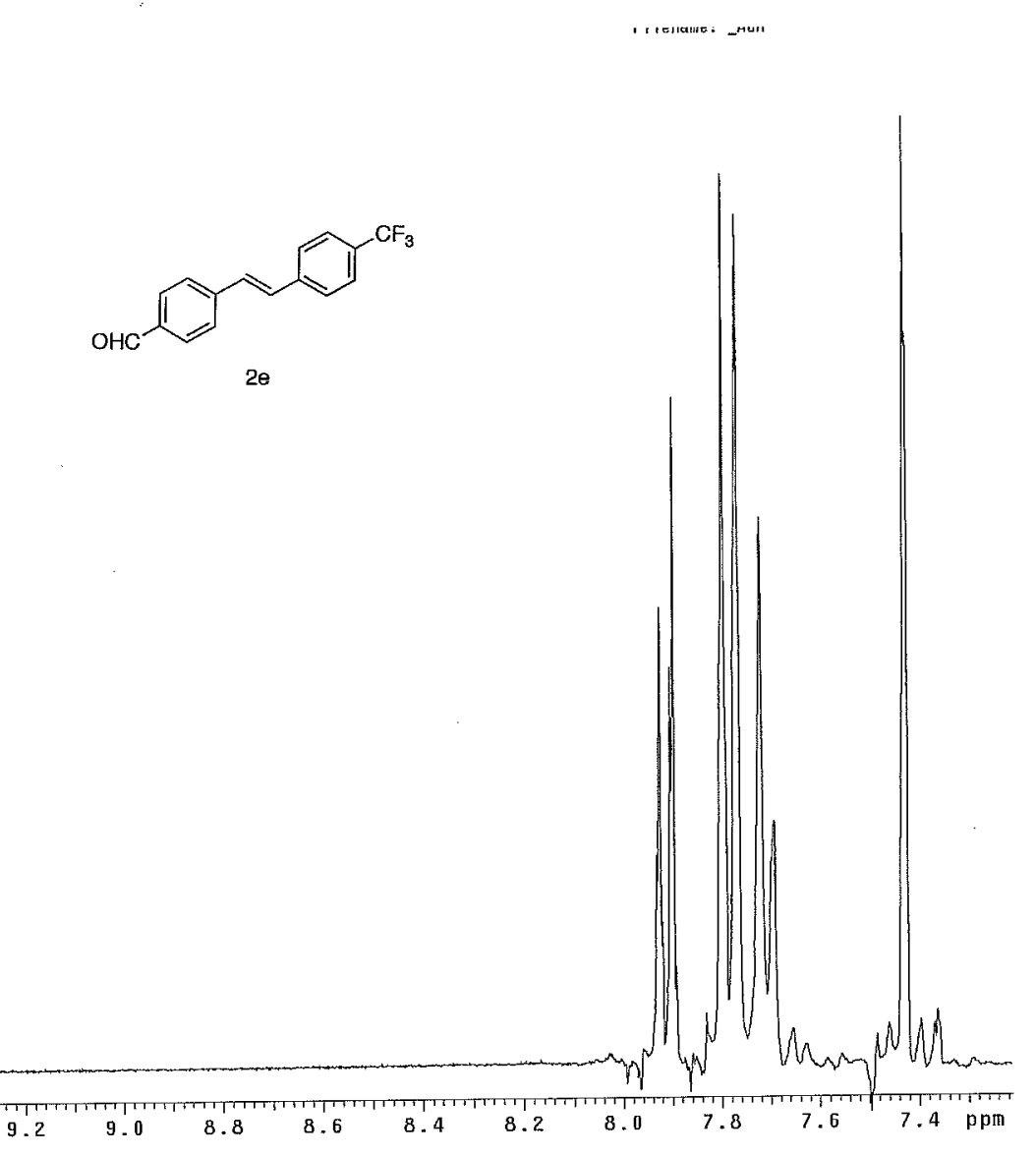

Pulse sequenco: s2pul

Solvent: CO3CN 298.1 File: APD ACHDACF 3st l bene
Hercury-300BB "jasmine"

lay $1.000 \mathrm{sec}$

$300.0546243 \mathrm{HH}$

Total time $0 \mathrm{~min}, 25 \mathrm{sec}$
To

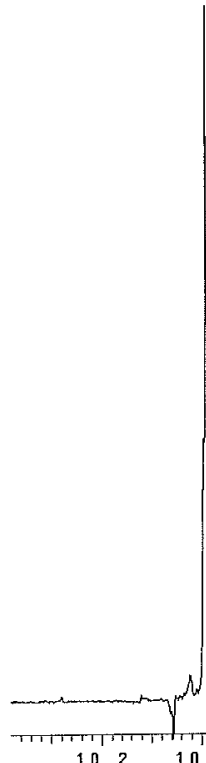

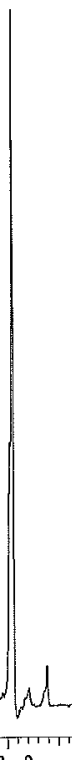

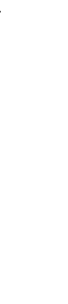

$\mathrm{OHC}$

Figure S11. Partial ${ }^{1} \mathrm{H}$ NMR spectrum of $2 \mathrm{e}\left(300 \mathrm{MHz}, \mathrm{CD}_{3} \mathrm{CN}\right)$. 
Relax. de lay $1.000 \mathrm{sec}$

Pulse 45.0 degrees

Width $18862.9 \mathrm{~Hz}$

12000 repetitions 4487184 MHZ

OECOUPLE H1, 300.4561560 MHZ

Power $37 \mathrm{~dB}$

cont i nuous $7 \mathrm{y}$ on

DATA PROCESSIMG

Line brozdening $1.0 \mathrm{~Hz}$

Total time 13 hr, 2 min, $10 \mathrm{sec}$
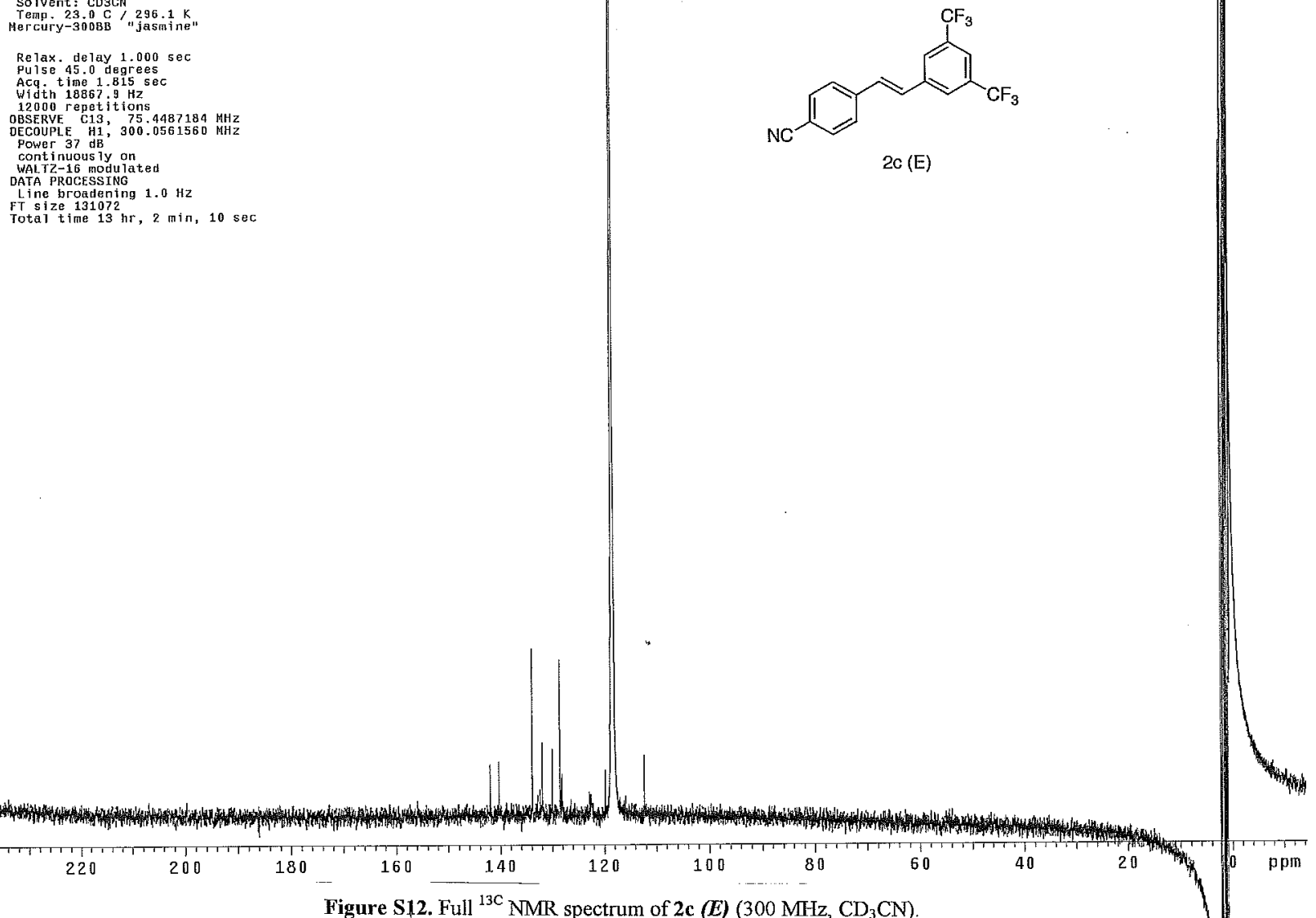

Archive directory: /export/home/unmr2/Ynmrsys/date File: CARBON

Pu1se sequence: s2pul

Solvent: cosch
Tespe $23.0 \mathrm{C} / 296-1 \mathrm{~K}$
Mercury-300B "Jasinine"

Relax. delay 1.000 sec

Pulse 45.0 degrees
Act. time 1.815 sec

Width $18867.9 \mathrm{~Hz}$

OBSERVE C13, 75.4487184
OECOUPL H1,
OE0.0561560 MHZ

continuous ly on

WALTL-16 modulated
DATA PROCESSHM

Line broadening $1.0 \mathrm{~Hz}$

FT size 131072 tim 2 min, 10 sec

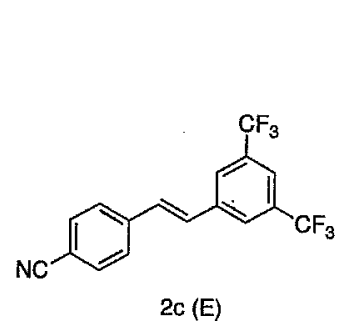

Figure S12. Full ${ }^{13 \mathrm{C}} \mathrm{NMR}$ spectrum of $2 \mathrm{c}(E)\left(300 \mathrm{MHz}, \mathrm{CD}_{3} \mathrm{CN}\right)$

$2 c(E)$

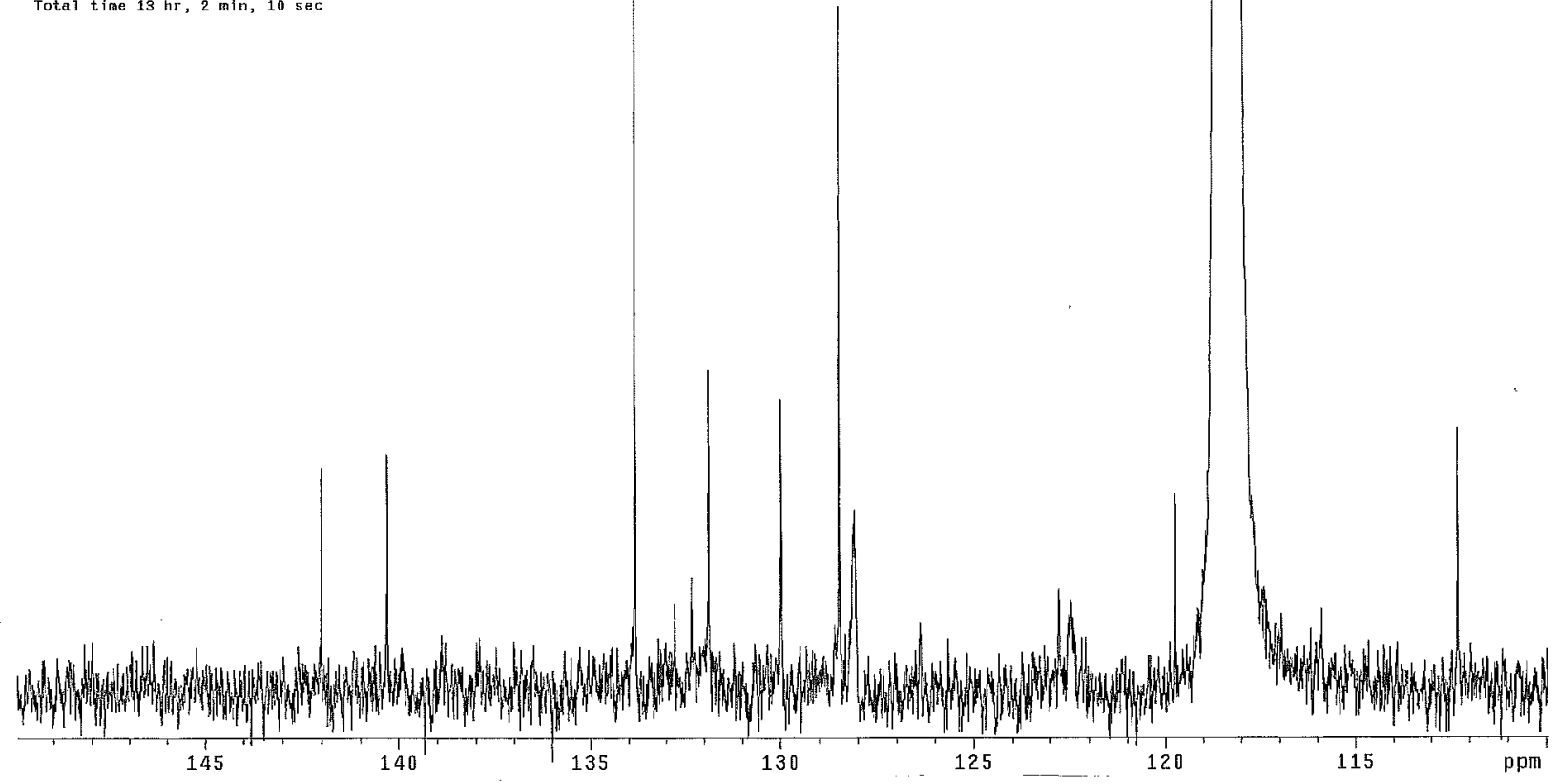

Figure S13. Partial ${ }^{13} \mathrm{C} \mathrm{NMR}$ spectrum of $2 \mathrm{c}(E)$ (300 MHz, $\mathrm{CD}_{3} \mathrm{CN}$ ). 
Pulse Sequence: s2pul Solvent: CD3CN Amblent temperature
Hercury-300BB "Jasmine"

Relax. de lay 1.000 sec

Acq. $t$ time 1.815 sec

Width 18867 it

OBSERVE C13, $75.4487219 \mathrm{MHZ}$
DECOUPLE H1, $300.0561560 \mathrm{MHZ}$

Power $37 \mathrm{~dB}$.

WALTZ-16 modu Tated

LAne brodening $1.0 \mathrm{~Hz}$

FT size 13102
Jotaltime $\mathrm{hr}, 42 \mathrm{~min}, 31$ sec

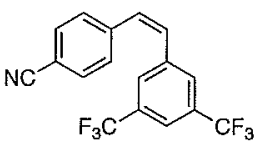

2c (Z)

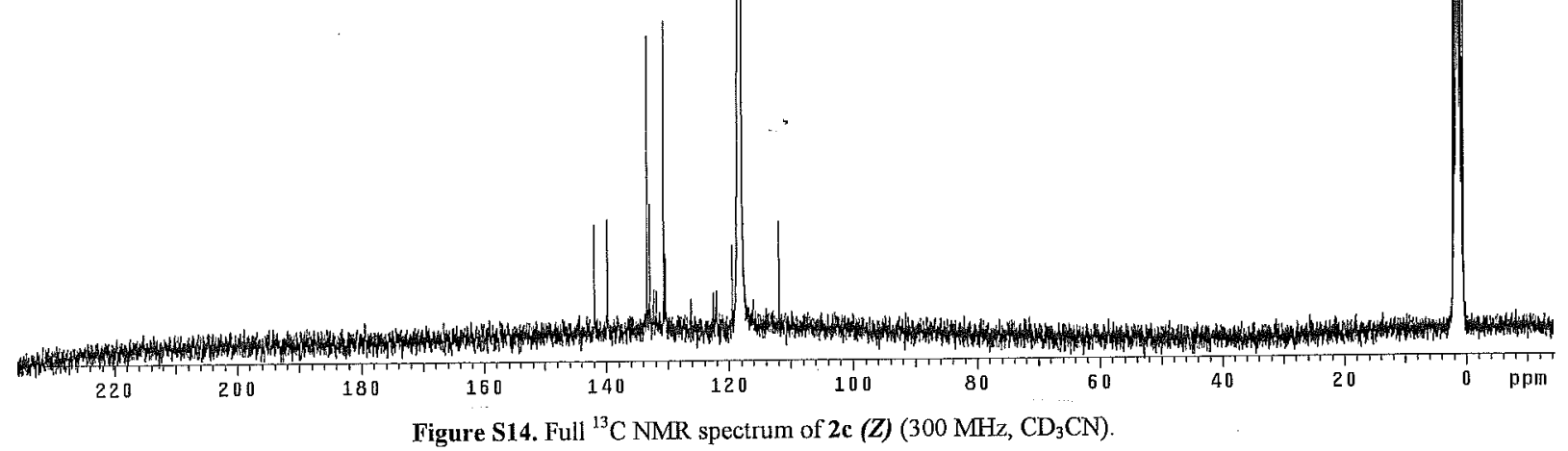

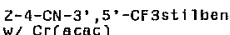

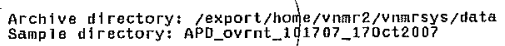

Pu1se Sequence: s2pu1

Solvent: CD3CN

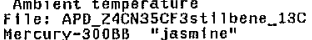

Re lax. delay $1.000 \mathrm{sec}$

ACᄃ. $t$ 1me 1.315 sec

Width 18867 is HZ

OBSERE CEB, 75.4487643 MHZ
OECOPLE HL, $300.0561560 \mathrm{MHZ}$

continuously on

WALTZ-16 modulated

DATA precssing 1.0

FT size 131072
Total time g hr, 42 min, $31 \mathrm{sec}$
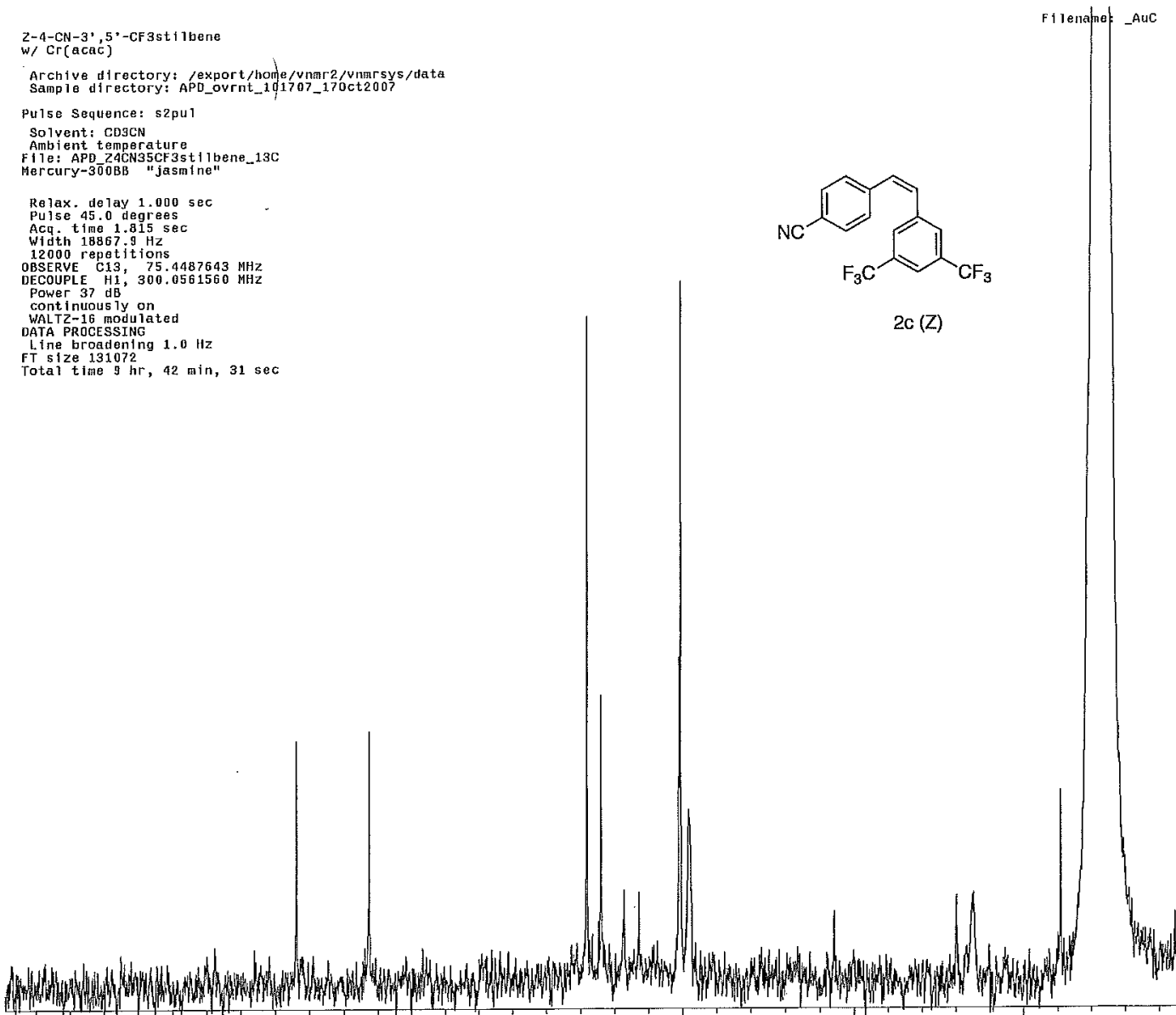

145140

135

130

120

Figure S15. Partial ${ }^{13} \mathrm{C}$ NMR spectrum of $2 \mathrm{c}(Z)\left(300 \mathrm{MHz}, \mathrm{CD}_{3} \mathrm{CN}\right)$ 
Pulse Sequence: szput

Solyent: $\mathrm{CD} 3 \mathrm{CN}$

Ambient temperature
File: APO 0 ct 182007

Relax de lay 1.000500

Pulse 45.0 degrees

Acq ith time 1.8067 .

DECOUPLE H1, 300.0561560 HHZ

Power $37 \mathrm{~dB}$

cont fnuous $7 y$ on

DATA PROCESSTMG

Line broadening $1.0 \mathrm{~Hz}$

FT $512 e 131072$
Total time $2 \mathrm{hr}, 45 \mathrm{~min}, 6 \mathrm{sec}$<smiles>O=Cc1ccc(/C=C/c2cc(C(F)(F)F)cc(C(F)(F)F)c2)cc1</smiles>

$2 d(E)$

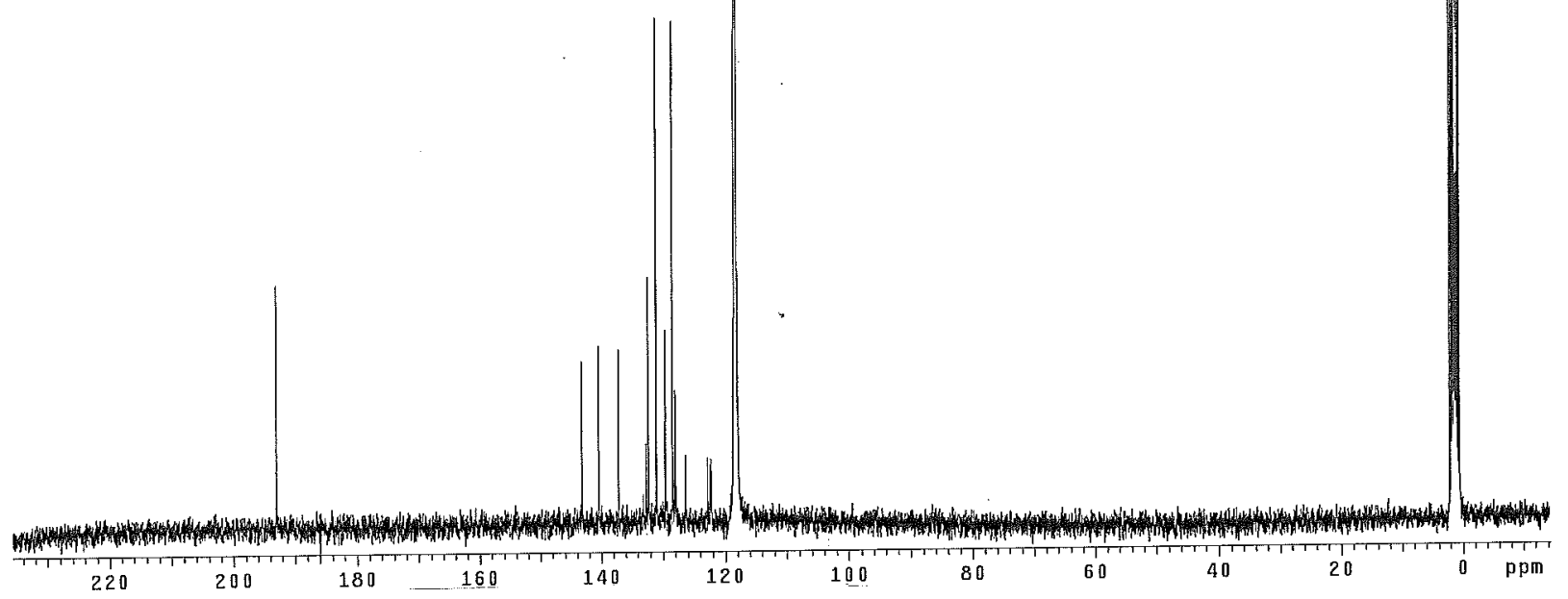

Figure S16. Full ${ }^{13} \mathrm{C}$ NMR spectrum of $2 \mathrm{~d}(\boldsymbol{E})\left(300 \mathrm{MHz}, \mathrm{CD}_{3} \mathrm{CN}\right)$.

E-4-CHO-3', 5'-CF3stilbene

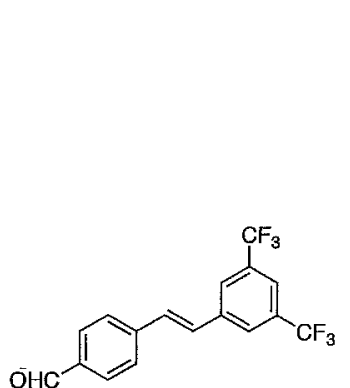

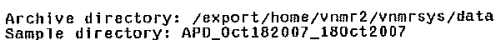

Pulse sequence: s2pui

Solvent: CDSCN

Ambient temperature
Fle: APD Det 182007
Mercury-300B ijasmine

Relax. delay $1.000 \mathrm{sec}$

Acq t time 1.815 sec

OBSERVE C13, 75.4487196 MHZ

Power $37 \mathrm{~dB}$, 30

cont inuous $1 y$ on
WALTZ-16 modulated

DATA ProcessiNG $1.0 \mathrm{~Hz}$

FT size 131072
Total time $2 \mathrm{hr}, 45 \mathrm{~min}, 6$ sec

2d (E)

Firename: AuC

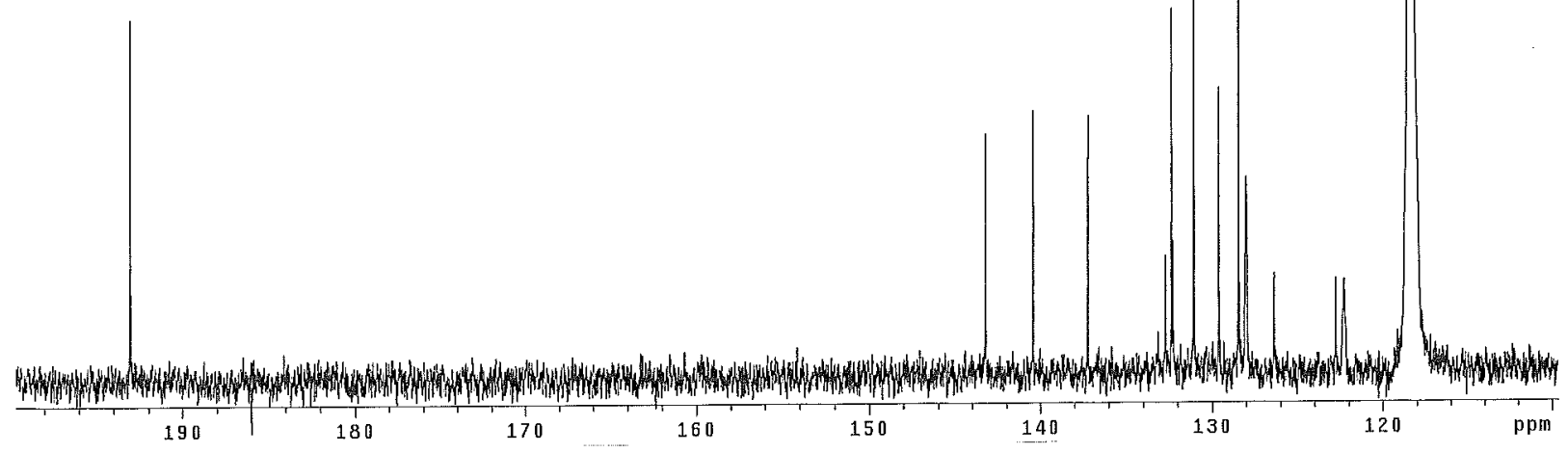

Figure S17. Partial ${ }^{13} \mathrm{C}$ NMR spectrum of $2 \mathrm{~d}(E)\left(300 \mathrm{MHz}, \mathrm{CD}_{3} \mathrm{CN}\right)$ 

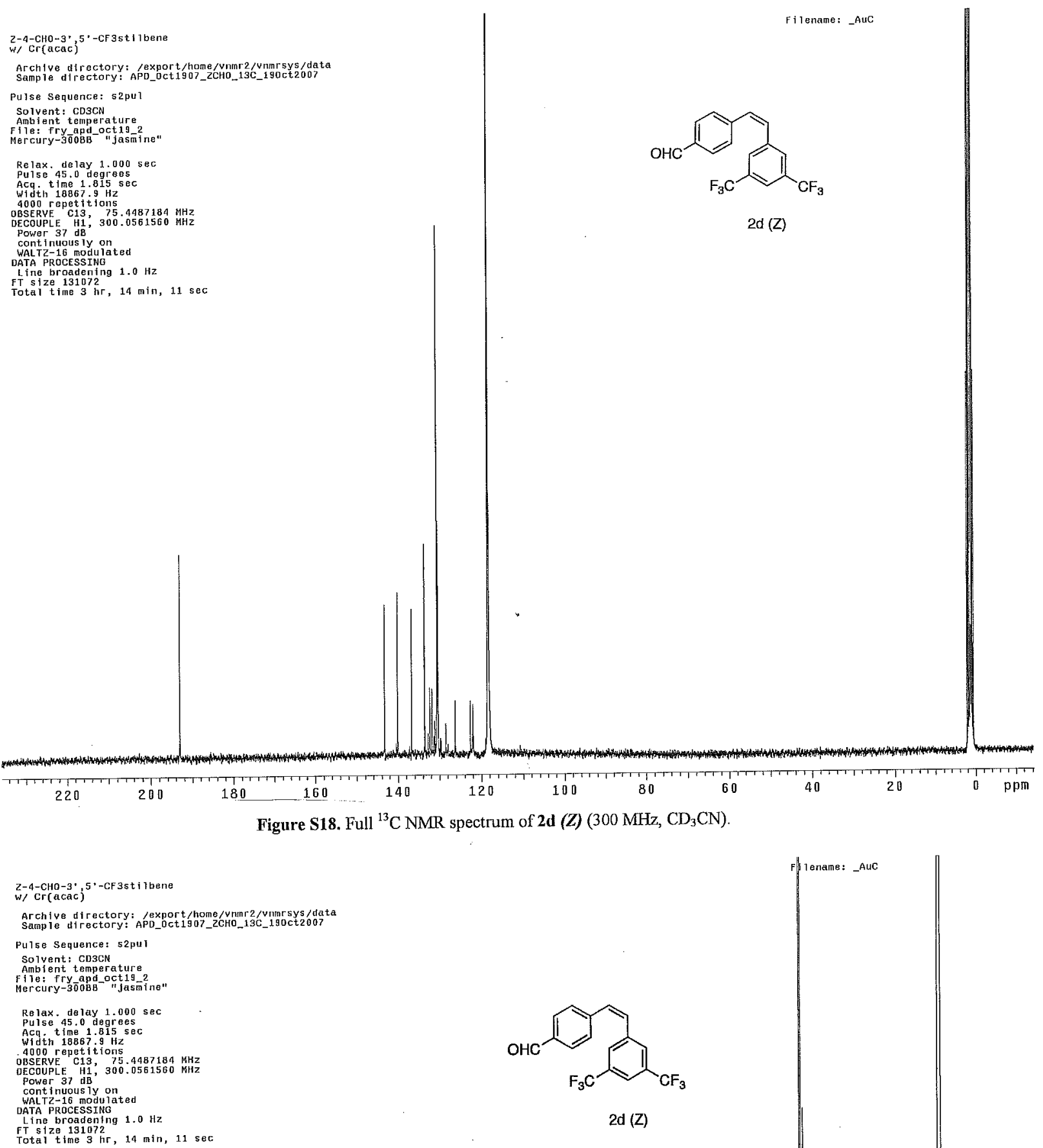

$2 \mathrm{~d}(\mathrm{Z})$
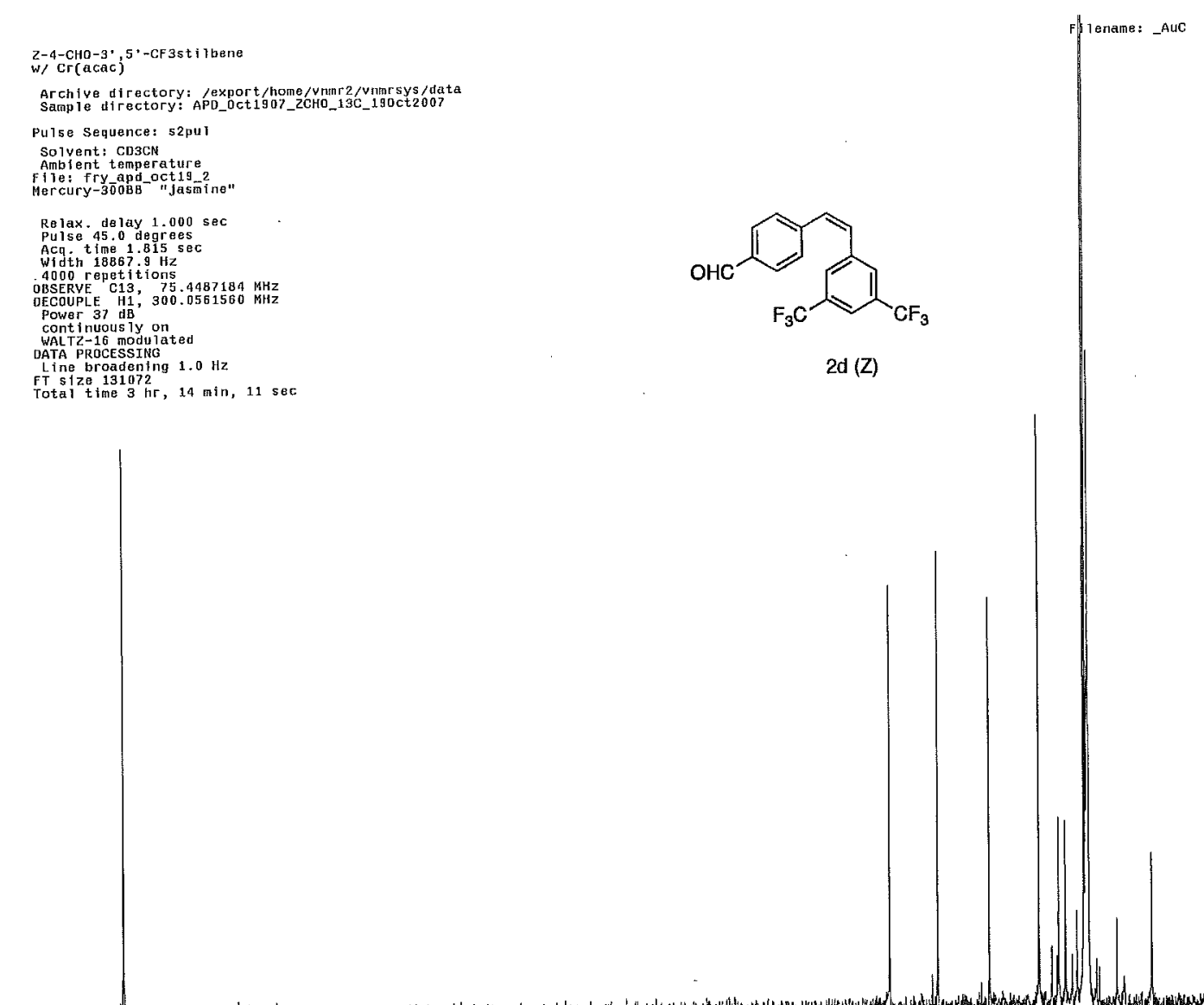

Figure S19. Partial ${ }^{13} \mathrm{C}$ NMR spectrum of $2 \mathrm{~d}(Z)\left(300 \mathrm{MHz}, \mathrm{CD}_{3} \mathrm{CN}\right)$. 
Pulse Sequence: szpul

Solvent: COSCN

Ambient temperature

Re lax. de lay $1.000 \mathrm{sec}$

Width $18867.9 \mathrm{HZ}$

OBSERYE C13, 75.4987637 MHI

DECOUPLE H1, 300.0561560 MHZ

Power $37 \mathrm{~dB}$

cont

DATA PROCESSING

The $1.0 \mathrm{~Hz}$

Total time $1 \mathrm{hir}, 37 \mathrm{~min}, 8 \mathrm{sec}$

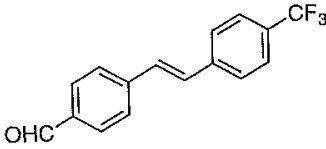

$2 \theta$

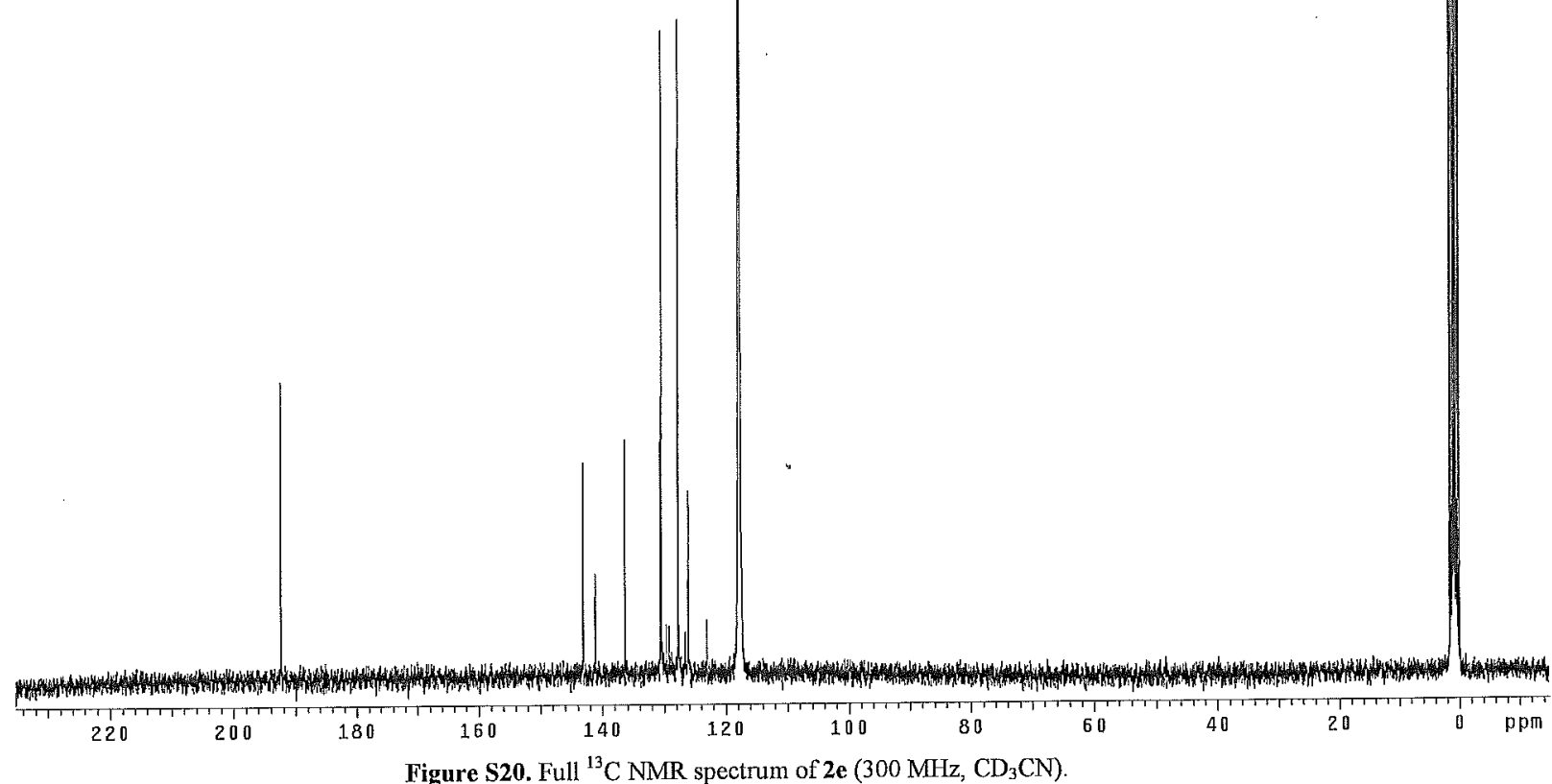

Figure S20. Full ${ }^{13} \mathrm{C}$ NMR spectrum of $2 \mathrm{e}\left(300 \mathrm{MHz}, \mathrm{CD}_{3} \mathrm{CN}\right)$,

4-CHO-4-CF3stilben

Filename: AUC

Archive directory: /export/home/vinn 2/vnmisys/data

Pulse Sequence: s2pur

Solvent: $\mathrm{CD} 3 \mathrm{CN}$

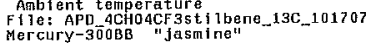

Relax. delay $1.000 \mathrm{sec}$

Acq. time 1.815 sec

With 18866 .

OBSERYE C13, 75.4487213 MIIZ

DECOUPLE H1; 3 OO.

Power $37 \mathrm{~dB}$, on
Continuous $1 \mathrm{y}$ on
WALTZ-16

DATA PROCESSING

FT size 131072
Total time $1 \mathrm{hr}, 37 \mathrm{~m} / \mathrm{h}, 85 \mathrm{ec}$
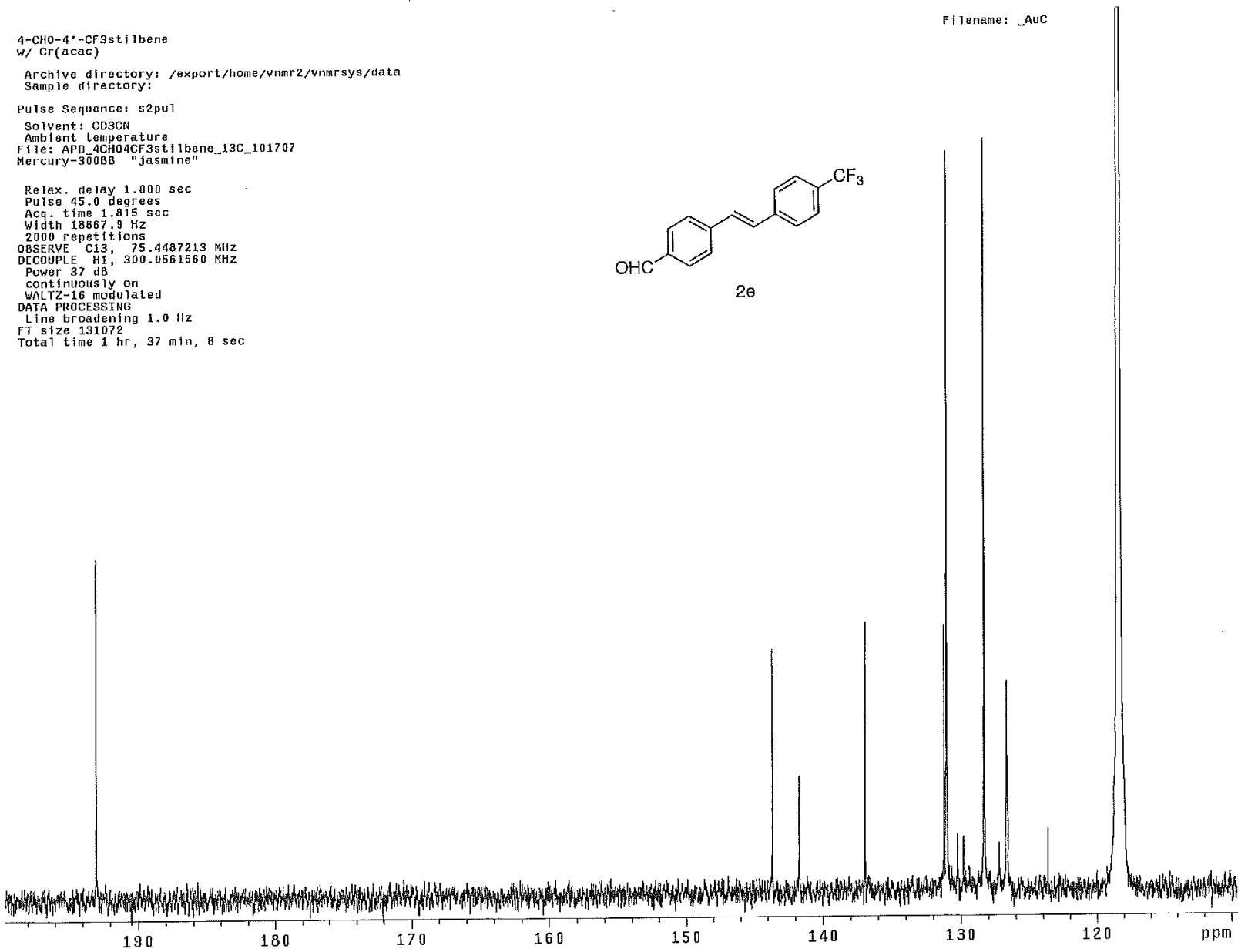

Figure S21. Partial ${ }^{13} \mathrm{C} N \mathrm{NR}$ spectrum of $2 \mathrm{e}\left(300 \mathrm{MHz}, \mathrm{CD}_{3} \mathrm{CN}\right)$. 


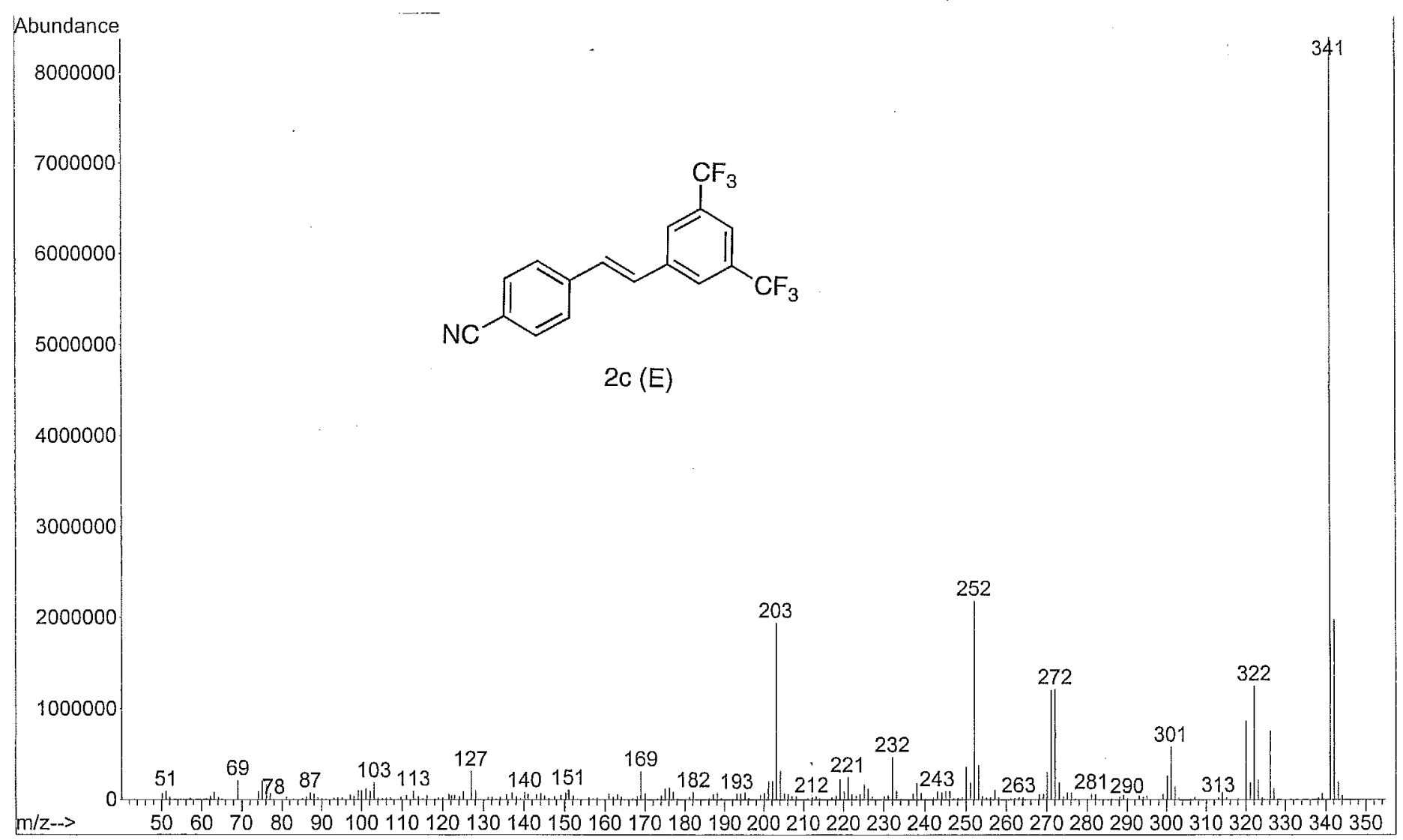

Figure S22. Representative mass spectrum of $2 \mathrm{c}(E$ and $Z$ ).

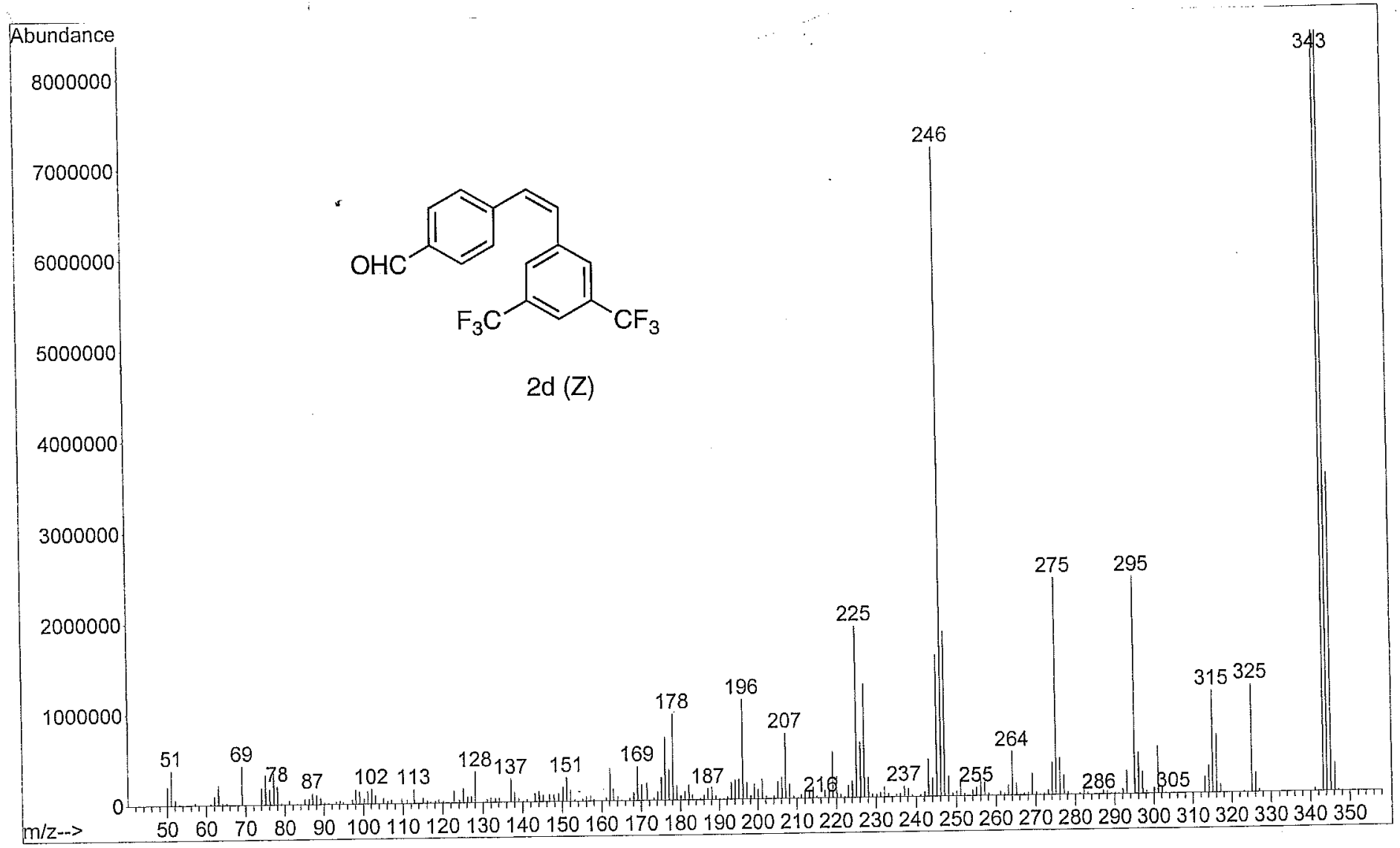

Figure S23. Representative mass spectrum of $2 \mathrm{~d}(E$ and $Z)$. 


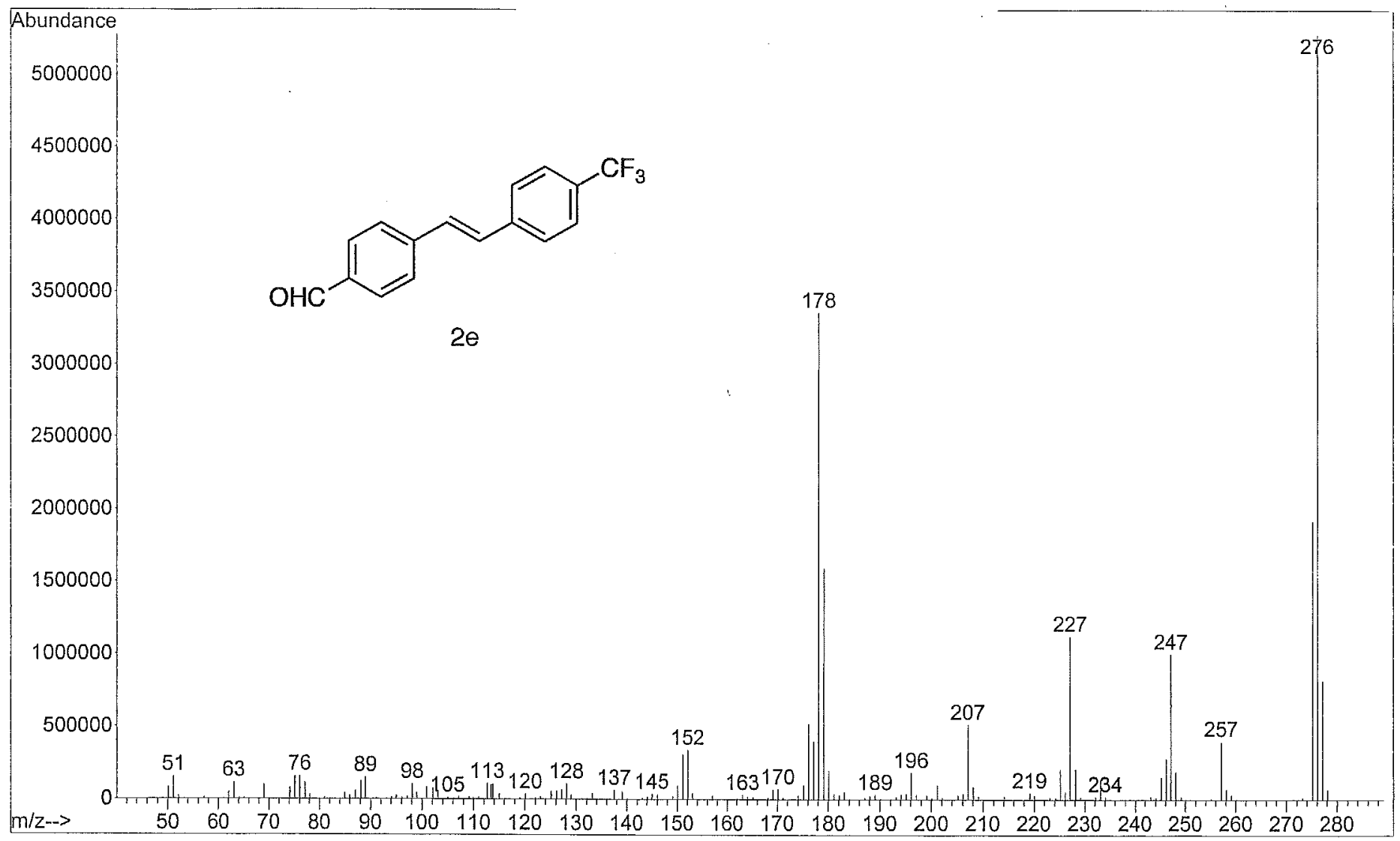

Figure S24. Mass spectrum of 2e. 